\title{
Automated Assignment of Simulated and Experimental NOESY Spectra of Proteins by Feedback Filtering and Self-correcting Distance Geometry
}

\author{
Ch. Mumenthaler ${ }^{1}$ and W. Braun ${ }^{2 *}$
}

${ }^{1}$ Institut für Molekularbiologie und Biophysik Eidgenössische Technische HochschuleHönggerberg, CH-8093 Zürich, Switzerland

${ }^{2}$ Department of Human Biological Chemistry \& Genetics, NMR Center University of Texas Medical Branch, Galveston, TX 77555-1157, USA
A new method for automatically assigning proton-proton NOESY spectra is described and demonstrated for simulated and experimental spectra of the proteins dendrotoxin $\mathrm{K}, \alpha$-amylase inhibitor tendamistat and the DNA-binding domain of the 434 repressor protein. The method assigns the NOESY spectrum and calculates three-dimensional protein structures simultaneously, using a list of proton chemical shifts and ${ }^{3} J_{\mathrm{NH} \alpha}$ coupling constants. An ensemble of structures is iteratively calculated by self-correcting distance geometry from unambiguous and selected ambiguous NOESY cross peaks. New structure based filters recognize the correct constraints from the ambiguous cross peak list. For the first round of assignment neither a preliminary initial structure nor a sufficient set of unambiguous NOESY cross peaks is needed. The method can also be applied to cross peak lists containing hundreds of noise peaks. For an assumed tolerance of $+/-0.01 \mathrm{ppm}$ in the chemical shifts of the peak positions, only about $10 \%$ of the NOESY cross peaks can be unambiguously assigned based on their chemical shifts alone. Our automated method assigned about $80 \%$ of all cross peaks with this chemical shift tolerance, and 95 to $99 \%$ of the assignments were correct. The average pairwise RMSD for the backbone atoms of the ten best final structures is about $1.5 \AA$ in all three proteins and the previously determined NMR solution structures are always embedded in this structure bundle. We regard our method as a highly practical tool for automatic calculation of three-dimensional protein structures from NMR spectra with minimal human interference.

(C) 1995 Academic Press Limited

Keywords: NMR; automated assignment; distance geometry in torsion angles; three-dimensional solution structure
1982), assignment of two-dimensional homonuclear (Wagner \& Wüthrich, 1982) or three- and fourdimensional heteronuclear edited NOESY spectra (Fesik \& Zuiderweg, 1988; Clore \& Gronenborn, 1991) and the calculation of three-dimensional structures from distance constraints (Braun et al., 1981, 1983; Havel et al., 1983; Clore et al., 1985; Braun \& Gō, 1985). Several strategies for sequence specific assignments by combining various heteronuclear experiments have been proposed (Bax \& Grzesiek, 1993), which lead to complete assignments of proteins with molecular weight up to $30 \mathrm{kDa}$ (Fairbrother et al., 1992; Stockmann et al., 1992; Grzesiek et al., 1992; Thériault et al., 1993; Spitzfaden et al., 1994; Fogh et al., 1994; Remerowski et al., 1994). Some of these sequence specific assignments methods have been automated in 
recent years (Hare \& Prestegard, 1994; Zimmermann et al., 1994; Olson \& Markley, 1994; Morelle et al., 1995; Bartels, 1995).

The successful automated sequence specific assignment is, however, only one step in the chain of computerized tools from the experimental NMR spectra to the three-dimensional structure of proteins. Even with a complete assignment of all nuclei, one still has to extract distance constraints from NOESY spectra by assigning all or almost all peaks in these spectra. This part of the structure determination is time consuming and error-prone. The accuracy of determining the peak positions is limited by the linewidths, the differences of the chemical shifts observed in J-correlated and NOESY spectra, and by overlap of cross peaks in homonuclear ${ }^{1} \mathrm{H}^{1} \mathrm{H}$ NOESY spectra. Heteronuclear ${ }^{15} \mathrm{~N}$ or ${ }^{13} \mathrm{C}$ correlated ${ }^{1} \mathrm{H},{ }^{1} \mathrm{H}$ NOESY spectra have significantly increased the limit for the size of proteins that can be studied by the NMR technique, but overlap of cross peaks occurs also in these spectra for large proteins (Wagner, 1993; Gronenborn \& Clore, 1994).

Recently, new approaches were proposed to combine three-dimensional structure calculations more tightly with the assignment problem (Oshiro \& Kuntz, 1993; Kraulis, 1994). In these approaches, the spatial positions of ${ }^{1} \mathrm{H}$ nuclei are calculated without assigning them to specific protons in the polypeptide chain, and the ${ }^{1} \mathrm{H}$ nuclei are then assigned on the basis of their relative spatial positions. These studies had some success with simulated data sets, assuming that chemical shifts are known with unlimited accuracy and uniquely identify ${ }^{1} \mathrm{H}$ nuclei. In a different semiautomatic procedure (Meadows et al., 1994) NOESY spectra were iteratively assigned in combination with calculation of three-dimensional structures. The overlap problem was completely ignored in these studies. This problem of calculating threedimensional structures with ambiguous data was successfully approached by Nilges (1995) in a simulated annealing method, where the restraints from all ambiguous assignments were included as a $r^{-6}$ weighted sum in the NOE target function.

We present a new, completely different approach to this problem, based on the "self-correcting distance geometry" method (Hänggi \& Braun, 1994). This method was improved and used to predict the correct fold of small $\alpha$-helical proteins (Mumenthaler \& Braun, 1995). We perform distance geometry calculations in torsion angles (Braun \& Gō, 1985; Güntert et al., 1991) with distance constraints from unambiguous and from some ambiguous peaks. New structure based filters recognize correct constraints in a list of ambiguous cross peaks. This ambiguity arises because the chemical shifts of the cross peaks and their corresponding protons coincide only within a tolerance range which we varied from $0.01 \mathrm{ppm}$ to $0.03 \mathrm{ppm}$. Chemical shift variations of the order of $0.01 \mathrm{ppm}$ are typically found in protein spectra. In iterative cycles of automated spectral analysis and

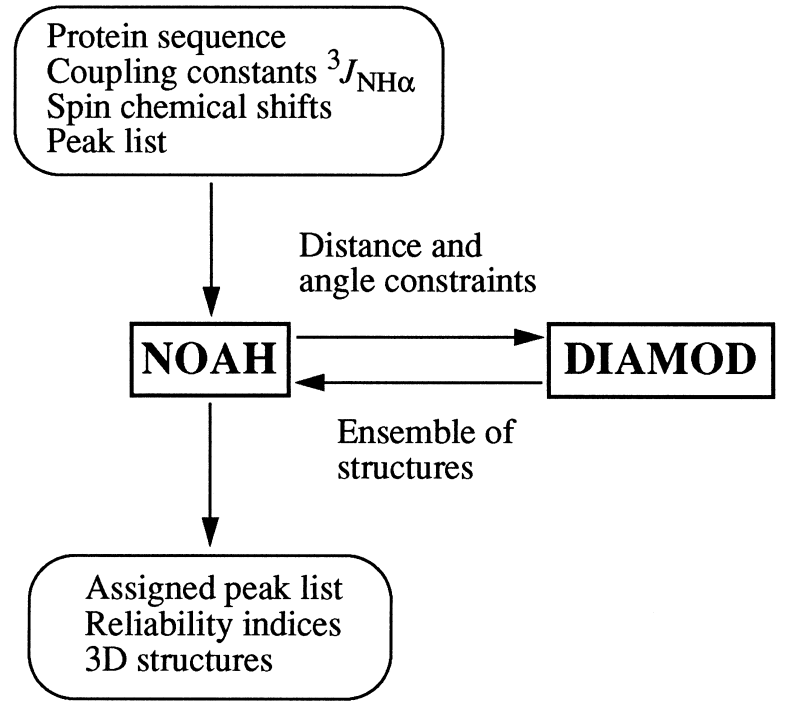

Figure 1. Data flow and relation between the programs NOAH and DIAMOD in the automated assignment of NOESY spectra. Input and output files of the procedure are written in rounded boxes.

three-dimensional structure calculations, an increasing number of correct assignments are identified.

We applied our automatic assignment method to simulated and experimental NOESY cross peak lists for three proteins and showed that the accuracy of this method is sufficient to determine the threedimensional structures with a high accuracy and without bias. We also introduce a new quantity, the reliability distance (RD), to measure the reliability of the individual assignments. In practice, the work required to assign NOESY spectra is dramatically reduced by applying our automatic method.

\section{Automated Assignment of NOESY Spectra}

\section{Overview on programs and data files}

Our assignment method requires as input data: the amino acid sequence of the protein, a nearly complete list of protons with their chemical shifts $\omega$, a list of ${ }^{3} J_{\mathrm{NH} \alpha}$ coupling constants and a proton NOESY cross peak list with cross peak positions $\left(\omega_{1}, \omega_{2}\right)$ and their NOE intensities (Figure 1). These input data are analysed by the program NOAH, which generates dihedral angle constraints from the ${ }^{3} J_{\mathrm{NH} \alpha}$ coupling constants and distance constraints from a subset of NOESY cross peaks selected by the extent of their ambiguity. The distance geometry program DIAMOD (Hänggi \& Braun, 1994; Mumenthaler \& Braun, 1995) calculates an ensemble of three-dimensional protein structures from these constraints, starting from randomly generated structures. Because the assignment of many NOESY cross peaks are ambiguous (i.e. several possible proton pairings could account for it) a substantial 
Table 1. Simulated and experimental data sets used to test the efficiency and reliability of the automated assignment method

\begin{tabular}{|c|c|c|c|c|c|c|}
\hline \multirow[b]{2}{*}{ Protein } & \multirow[b]{2}{*}{ Code } & \multirow{2}{*}{$\begin{array}{c}\Delta_{\text {tol }^{\mathbf{a}}} \\
(\mathrm{ppm})\end{array}$} & \multirow{2}{*}{$\begin{array}{c}d_{\operatorname{sim}^{b}}{ }^{b} \\
(\AA)\end{array}$} & \multicolumn{2}{|c|}{ Peaks ${ }^{c}$} & \multirow{2}{*}{$\begin{array}{c}N_{\text {unamb }}{ }^{\mathrm{d}} \\
(\%)\end{array}$} \\
\hline & & & & $P_{\text {tot }}$ & $P_{\text {uа }}$ & \\
\hline \multirow{5}{*}{ Dendrotoxin K } & DEN1 & 0.01 & 4.0 & 1847 & 1466 & 9.9 \\
\hline & DEN2 & 0.02 & 4.0 & 1847 & 1466 & 2.2 \\
\hline & DEN3 & 0.03 & 4.0 & 1847 & 1466 & 1.0 \\
\hline & DENa & 0.02 & 5.0 & 3254 & 2654 & 2.2 \\
\hline & DENb & 0.02 & 5.0 & 1847 & 1498 & 2.6 \\
\hline \multirow[t]{2}{*}{ Tendamistat } & TEN1 & 0.01 & 4.0 & 1967 & 1546 & 10.2 \\
\hline & TEN2 & 0.02 & 4.0 & 1967 & 1546 & 3.8 \\
\hline \multirow[t]{2}{*}{ P434 repressor } & REP1 & 0.01 & 4.0 & 2048 & 1618 & 10.4 \\
\hline & REP2 & 0.02 & 4.0 & 2048 & 1618 & 3.0 \\
\hline \multirow[t]{2}{*}{ Dendrotoxin K } & NMRa & 0.01 & - & 1194 & 876 & 15.5 \\
\hline & $\mathrm{NMRb}$ & 0.01 & - & 1194 & 1194 & 19.5 \\
\hline
\end{tabular}

\footnotetext{
${ }^{a}$ Chemical shift tolerance used during the NOAH cycles. All protons within $+/-\Delta_{\text {tol }}$ of the cross peak chemical shift were considered as possible assignment candidates.

${ }^{b}$ Cutoff distance used for the simulation of the NMR spectrum. A cross peak was generated between all protons that were within a distance of $d_{\text {sim }}$ in the NMR structure. Pseudo-atom corrections were added to $d_{\text {sim }}$ if necessary

${ }^{\mathrm{c}}$ In most of the data sets, we assumed that the assignments of the sequential cross peaks were known. Therefore, we give the total number of peaks $\left(P_{\text {tot }}\right)$ and the total number of unassigned peaks $\left(P_{\text {ua }}\right)$ in the spectrum.

${ }^{d}$ Fraction of peaks that can be unambiguously assigned when using a tolerance range of $\Delta_{\text {tol }}$.
}

fraction of the distance constraints are not correct. The target function in DIAMOD has been designed to minimize the influence of these errors on the calculated structures (Hänggi \& Braun, 1994). DIAMOD returns these preliminary structures to $\mathrm{NOAH}$, which uses them to lower the ambiguity of the cross peaks and to filter the ambiguous assignments from the previous cycle. After 20 to 30 cycles, the result is a set of three-dimensional structures and a peak list of proposed assignments.

\section{Simulated and experimental NOESY cross peak lists}

Automatic assignments of NOESY cross peak lists from three different protein folds were tested: dendrotoxin $\mathrm{K}$ from the venom of black mamba (Berndt et al., 1993), which is a mixed $\alpha / \beta$-protein with 57 residues, the $\alpha$-amylase inhibitor tendamistat (Kline et al., 1988), a $\beta$-sheet protein containing 74 residues, and the DNA-binding domain of the phage 434 repressor (Neri et al., 1992), which contains only $\alpha$-helices and has 69 amino acid residues (Table 1). We performed calculations for all three proteins with the chemical shift tolerance range $\Delta_{\text {tol }}$ of $0.01 \mathrm{ppm}$ and $0.02 \mathrm{ppm}$; DEN1, DEN2, TEN1, TEN2, REP1 and REP2. NOESY cross peak lists were simulated from the experimentally determined proton chemical shifts and from the NMR solution structures of these proteins, using a cutoff distance of $4 \AA$. We assumed that all sequential cross peaks, i.e. peaks between $\mathrm{H}^{\alpha}, \mathrm{HN}$, $\mathrm{H}^{\beta 1}$ and $\mathrm{H}^{\beta 2}$ of the same or neighbouring residues were already assigned as a result of the sequence specific assignment procedure. Approximately 2000 cross peaks in a $\mathrm{H}_{2} \mathrm{O}$ NOESY spectrum recorded with short mixing time are typical for proteins of this size. Less than $10 \%$ of the cross peaks are unambiguous if the chemical shift tolerance is set to $0.01 \mathrm{ppm}$ and only a few percent are unambiguous for $0.02 \mathrm{ppm}$.

Additional data sets were tested for dendrotoxin $\mathrm{K}$. We wanted to study the performance of our method for an increased chemical shift tolerance of $0.03 \mathrm{ppm}$ (DEN3), for a different number of cross peaks (DENa and DENb), and for an experimental peak list (NMRa and NMRb). We used the final cross peak list of the experimental NOESY spectrum with mixing time $\tau_{\mathrm{m}}=40 \mathrm{~ms}$, which was recorded and analysed for the determination of the NMR solution structure of dendrotoxin K (Berndt et al., 1993). This list contained 1194 assigned peaks. In the data set NMRa we assumed that sequential cross peaks were already assigned. In data set $\mathrm{NMRb}$ no assignments of the cross peaks were retained to test the effect of this partial assignment on the result.

\section{Results}

We will first present the results of the NOAH calculations for the proteins dendrotoxin $\mathrm{K}$, tendamistat and 434 repressor. With a chemical shift tolerance range of $0.01 \mathrm{ppm}$, about $80 \%$ of the peaks were assigned for all three proteins. The percentage of wrongly assigned peaks was less than $5 \%$ in these calculations and the average pairwise RMSD of the final structure bundle was around $1.5 \AA$. The calculations for dendrotoxin $\mathrm{K}$ with the experimental $\mathrm{H}_{2} \mathrm{O}$ peak list achieved similarly good results. We also examine the progress of the whole procedure during the iterative cycles in more detail. Finally we show that our reliability checks permit the identification of a subset of assignments which are safe and that noise peaks do not disturb the method unduly.

\section{Accuracy of the automated assignment procedure}

Table 2 shows the extent and the accuracy of the automated assignment procedure for all calculations. Between 56 and $87 \%$ of the peaks were assigned for the data sets with a chemical shift tolerance of $\Delta_{\mathrm{tol}} \leqslant 0.02 \mathrm{ppm}$. The percentage of wrongly assigned peaks rises from 0.8 to $12.0 \%$ as the parameter $\Delta_{\text {tol }}$ increases. The calculations TEN1 and REP1 were particularly successful with less than $2 \%$ of wrongly assigned peaks. All calculations with $\Delta_{\text {tol }}=0.01 \mathrm{ppm}$ have low error rates of a few percent. In the automatic assignment of the experimental peak list of dendrotoxin K, NMRa and $\mathrm{NMRb}$, the error rates were similar to those in the simulated data set DEN1.

For structure calculations of proteins, the validity of the distance constraints is of prime importance. 
The distance constraints derived from the final assignment can be checked with the experimental NMR structure. $N_{\text {vio }}$ counts all violations of the constraints greater than $0.5 \AA$ in the NMR reference structure. In all calculations, including NMRa and $\mathrm{NMRb}$, the percentage of violating constraints is around $1 \%$ (Table 2). This is much lower than the percentage of the assignment errors. This difference between $N_{\text {err }}$ and $N_{\text {vio }}$ is due to the fact that at a chemical shift tolerance of $0.01 \mathrm{ppm}, 20$ to $30 \%$ of all NOESY cross peaks correspond to at least two different pairs of protons with a distance of less than $5 \AA$ (see below). This means that even with a narrow bundle of highly refined NMR structures one could not detect a large portion of the final wrong assignments.

When the chemical shift tolerance was raised to $\Delta_{\text {tol }}=0.03 \mathrm{ppm}$ (DEN3), less than $50 \%$ of the peaks were assigned. This result is partially due to the fact that the number of NOAH cycles was insufficient to reach convergence. We could significantly increase the extent of the assignments and decrease the RMSD values of the calculated structures by adding ten cycles to the schedule. Peaks with five and then with six different assignment possibilities were also taken into account during these ten additional cycles. The number of assigned peaks rose from 39 to $58 \%$ and the calculation ended with $N_{\text {err }}=16.5 \%$ and $N_{\text {vio }}=3.5 \%$. Even though these errors are higher than after the 26th cycle, the pairwise RMSD of the structures dropped from 3.1 to $1.7 \AA$ and the RMSD to the NMR structure decreased from the range 4.9 to $6.0 \AA$ to the range 2.1 to $2.6 \AA$.

Table 2. Extent and accuracy of the automated assignment procedure for the 11 data sets in the three proteins

\begin{tabular}{|c|c|c|c|c|c|c|c|}
\hline \multirow[b]{2}{*}{ Code } & \multirow{2}{*}{$\begin{array}{c}N_{\text {ass }}{ }^{\mathrm{a}} \\
(\%)\end{array}$} & \multirow{2}{*}{$\begin{array}{c}N_{\text {err }}^{b} \\
(\%)\end{array}$} & \multirow{2}{*}{$\begin{array}{c}N_{\mathrm{vio}}{ }^{\mathrm{c}} \\
(\%)\end{array}$} & \multirow{2}{*}{$\begin{array}{c}V i o_{\max }{ }^{\mathrm{d}} \\
(\AA)\end{array}$} & \multicolumn{3}{|c|}{$\operatorname{RMSD}(\AA)^{\mathbf{e}}$} \\
\hline & & & & & $\langle$ Ave $\rangle$ & Lowest & Highest \\
\hline DEN1 & 83 & 5.1 & 0.4 & 4.8 & 1.3 & 0.9 & 1.3 \\
\hline DEN2 & 65 & 8.1 & 0.9 & 4.0 & 1.7 & 1.2 & 2.1 \\
\hline DEN3 & 39 & 12.0 & 2.2 & 13.0 & 3.1 & 4.9 & 6.0 \\
\hline DENa & 68 & 6.0 & 0.7 & 2.9 & 0.7 & 0.6 & 0.8 \\
\hline $\mathrm{DENb}$ & 65 & 11.1 & 1.5 & 4.6 & 1.1 & 1.3 & 1.4 \\
\hline TEN1 & 87 & 0.8 & 0.1 & 0.7 & 1.7 & 1.3 & 2.0 \\
\hline TEN2 & 58 & 5.7 & 0.5 & 3.1 & 3.4 & 2.4 & 4.5 \\
\hline REP1 & 73 & 1.7 & 0.2 & 1.5 & 1.3 & 1.0 & 1.4 \\
\hline REP2 & 56 & 4.0 & 0.3 & 1.4 & 1.4 & 1.1 & 1.6 \\
\hline NMRa & 81 & 3.5 & 1.3 & 3.3 & 1.8 & 1.6 & 2.0 \\
\hline NMRb & 84 & 4.6 & 1.4 & 8.2 & 2.0 & 2.1 & 2.9 \\
\hline
\end{tabular}

a Number of final assignments as a fraction of $P_{\text {ua. }}$.

b Fraction of wrongly assigned cross peaks.

c Percentage of distance constraints derived from the final assignments that are violated in the NMR reference structure by more than $0.5 \AA$.

${ }^{d}$ Maximum violation of a constraint in the NMR reference structure. Constraints outside of the regions 2 to 56 (dendrotoxin), 5 to 73 (tendamistat) and 2 to 63 (repressor 434) were not considered, because the NMR reference structure is not representative outside these regions.

e The ten final structures with the lowest target function values were analysed. The pairwise average RMSD 〈ave〉, the lowest and the highest RMSD with respect to the NMR reference structure are given for the backbone atoms of the regions 2 to 56 (dendrotoxin), 5 to 73 (tendamistat) and 2 to 63 (repressor 434).

\section{Sampling and accuracy of the calculated structures}

The ten resulting structures with lowest target function values differ by an average pairwise backbone RMSD of less than $2 \AA$ in nine out of 11 calculations. With the exception of the calculation DEN3, which did not converge within the 26 cycles, the average pairwise RMSD of the calculated structures is similar to the RMSDs of the structures to the NMR reference structure. Thus, the NMR reference structure is embedded in the structure bundle. Figure 2 illustrates this good sampling property of our method for the calculations DEN1, TEN1 and REP1. The RMSD values obtained are typical for protein structures determined with data sets lacking stereospecific assignments and using a rather conservative calibration (Güntert et al., 1989).

By increasing the number of NOE cross peaks, as in the data set DENa compared to DENb, the RMSD values significantly dropped and came close to RMSD values of highly refined NMR structures (Clore et al., 1993). This agrees with the observation that the number of constraints is one of the main determinants for the precision of the structures. Automatic assignment methods might therefore be useful in the future to obtain a larger number of constraints in the refinement of structures.

Figure 3 illustrates the initial and the final stage of the calculation with the experimental peak list of dendrotoxin $\mathrm{K}$ (NMRa). The backbones of the ten best structures after the first cycle are shown in Figure 3(a). The folds of the structures do not resemble the native dendrotoxin $\mathrm{K}$ fold in this "embryo" stage of the assignment, but a consistent spatial separation of the loop region 14 to 19 and the C-terminal part is already manifest in all ten structures. This separation of regions, far apart also in the native fold, enables the program NOAH to discard some assignment possibilities in the next cycle. The mean structure of the final ten best structures, shown in blue together with the RMSD of the $\mathrm{C}^{\alpha}$ positions (red circles), is compared to the previously determined NMR structure (yellow) in Figure 3(b). Both backbone structures are within the root-mean-square deviations for almost all residues except for a short segment around residues 9 and 10 . As the unrefined structures, determined by our automatic assignment procedure, have been obtained without any manual assignment help, the good agreement with the previously determined structure (Berndt et al., 1993) shows the promising practical potential of our method.

\section{Quality of the assignments and distance constraints in the first NOAH cycles}

The first NOAH cycle is particularly difficult, because no preliminary structures are available to reduce the number of possible assignments. Figure 4(a) to (c) shows the occurrence of cross peaks with multiple assignments for the chemical shift tolerances of $0.01 \mathrm{ppm}, 0.02 \mathrm{ppm}$ and 
(a)
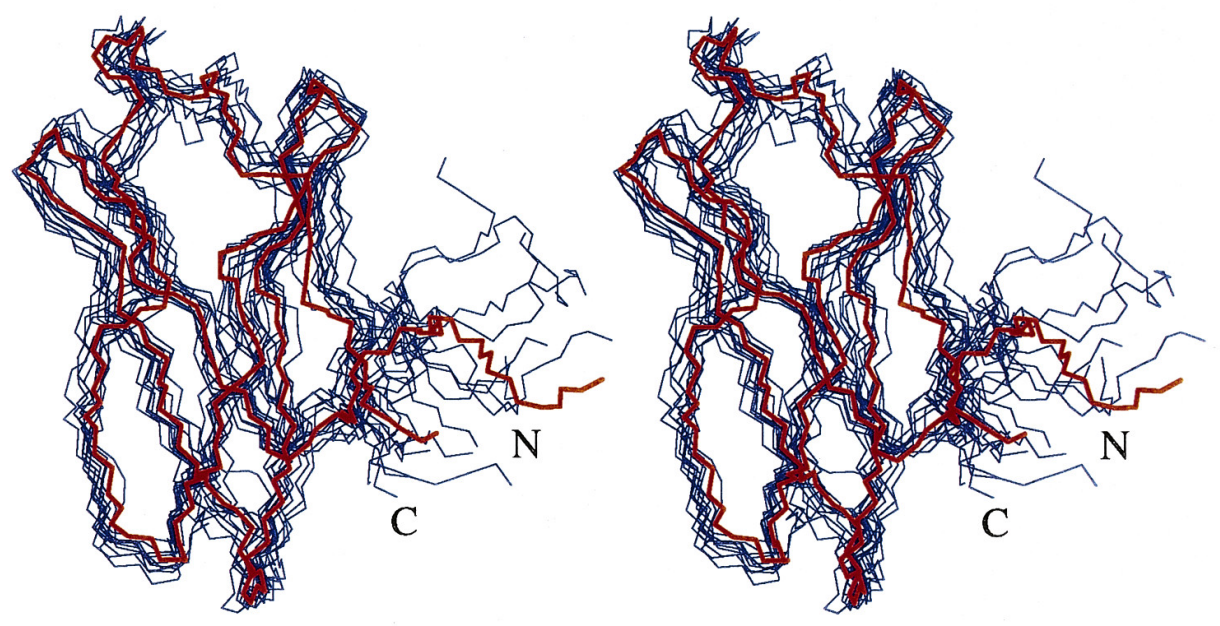

(b)
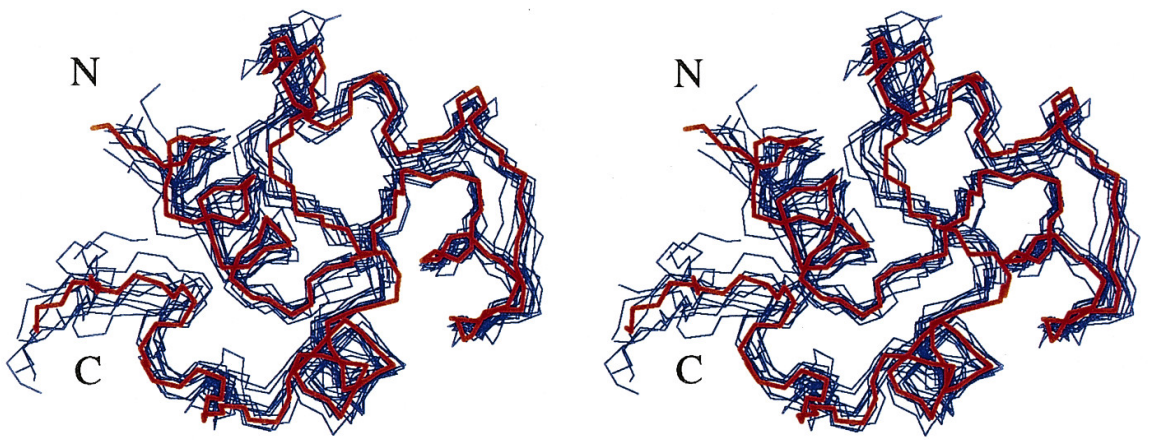

(c)
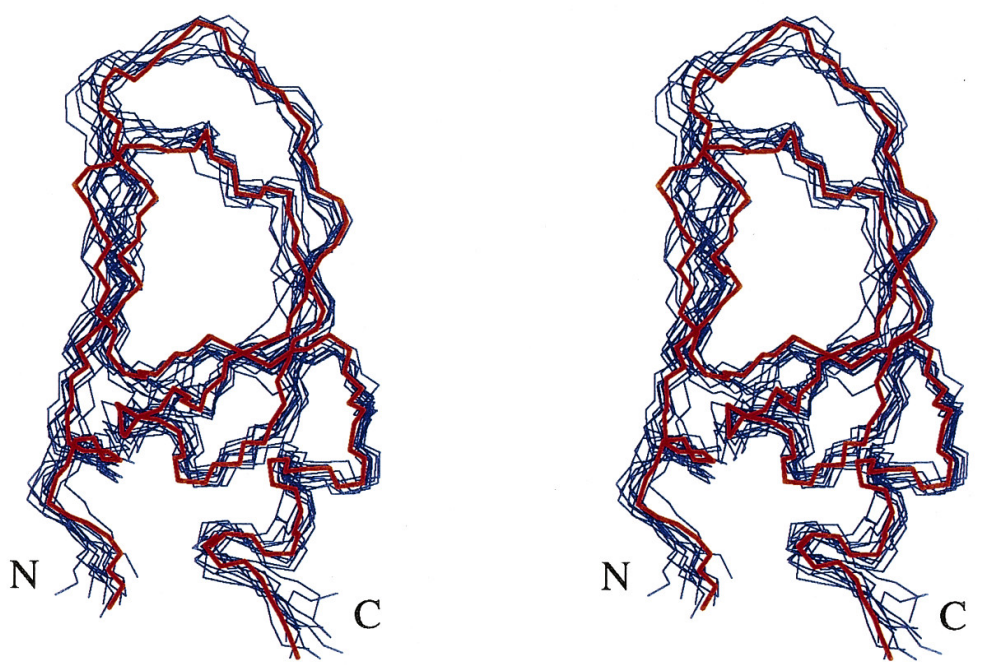

Figure 2. Superposition of the ten structures with lowest target function values obtained after the 25th NOAH cycle (blue) and the experimentally determined NMR structure (red) for the simulated data set (a) TEN1, (b) REP1 and (c) DEN1. The superposition was done on the backbone atoms of the residues 5 to 73 (TEN1), 2 to 63 (REP1) and 2 to 56 (DEN1). The flexible C-terminal loop of the repressor 434 between residues 64 and 69 is not shown.

$0.03 \mathrm{ppm}$, respectively. Only a small minority of the peaks, highlighted with black bars, can be unambiguously assigned from their chemical shifts alone. This coincides with observations made by Nilges (1995).
Besides unambiguous peaks we also include peaks with two assignment possibilities in the first NOAH cycle. This increases the number of peaks used in the DIAMOD calculation by a factor of 4 for DEN1 or 6 for DEN3, but a substantial fraction of 
the additional distance constraints are wrong (Table 3). Almost all of these wrong constraints are eliminated in the following cycles of selfcorrecting distance geometry and feedback filtering (Table 2).

\section{Improvements of the assignments and the calculated structures}

In Figure 5 the number of medium and long-range distance constraints derived from the

(a)

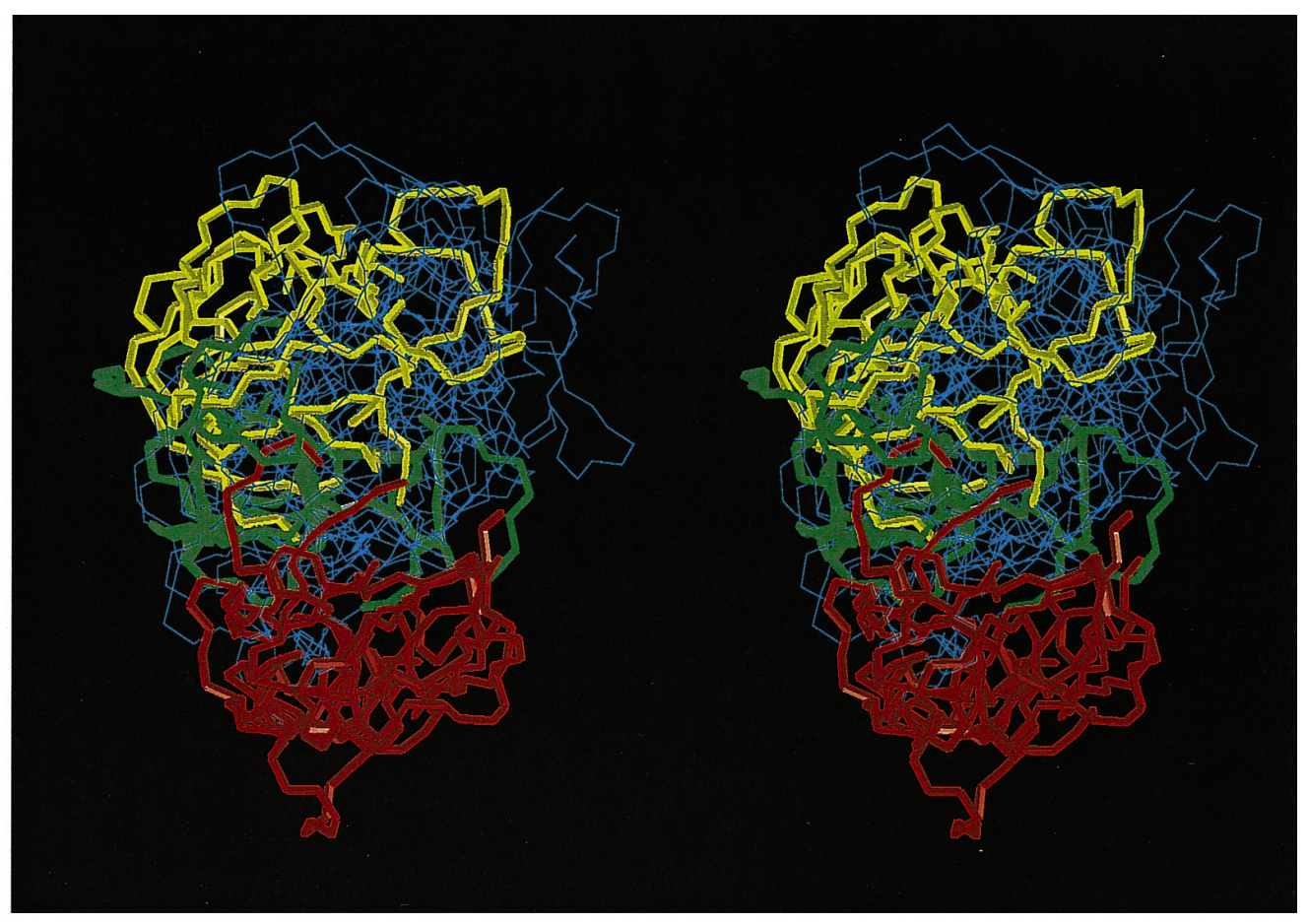

(b)

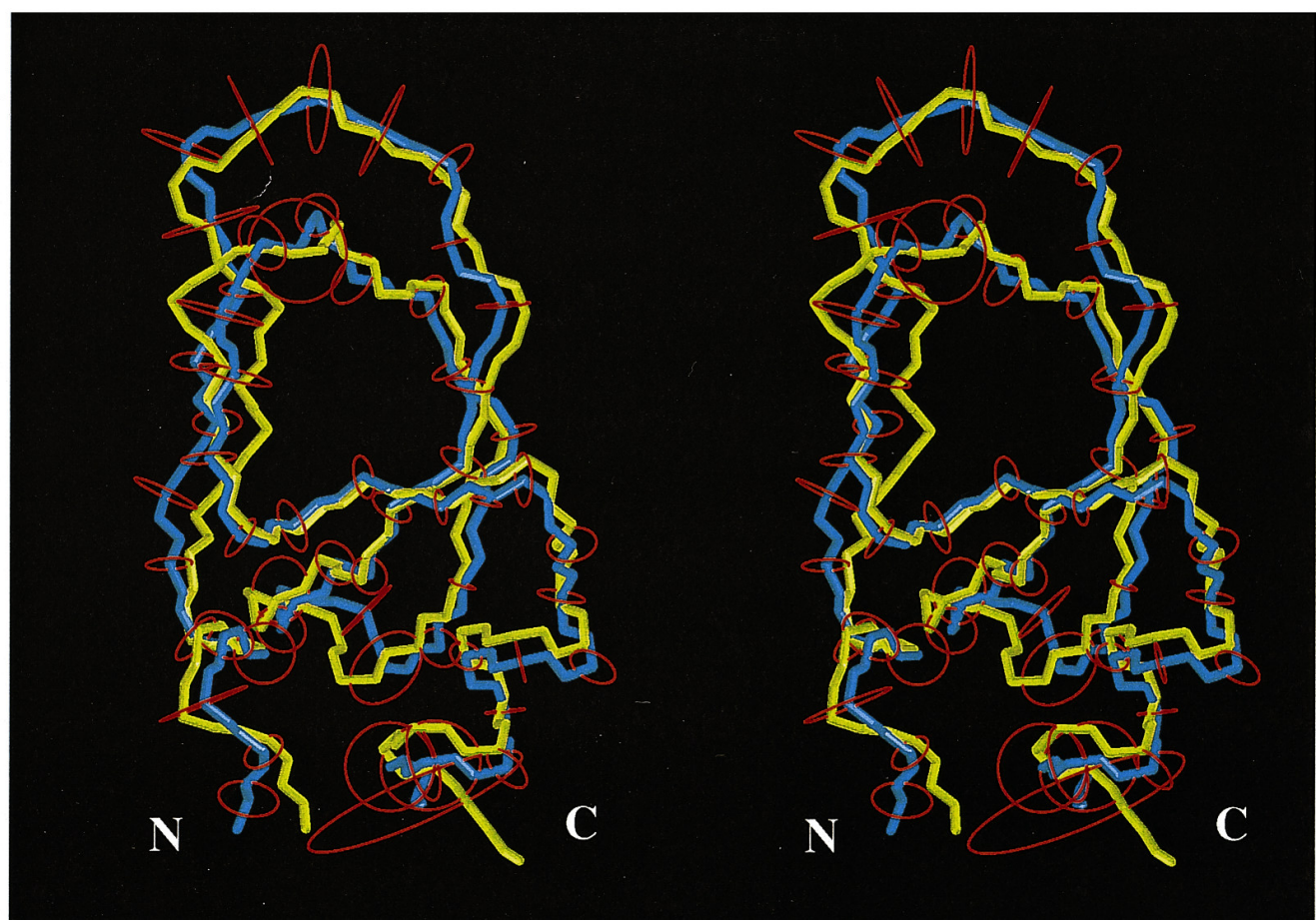

Figure 3. Stereoview of dendrotoxin $\mathrm{K}$ after the (a) first and (b) last assignment cycles of the calculation with the experimental peak list. (a) The ten structures with lowest target function values after the first cycle are superimposed on their backbone atoms of the region 2 to 56. The loop regions 14 to 19 and 36 to 40 as well as the C-terminal region 50 to 57 are colored in yellow, green and red, respectively. (b) The mean structure of the ten final structures with lowest target function values (blue) is superimposed on the NMR reference structure (yellow). The red circles indicate the mean RMSD of the $C^{\alpha}$ positions in the ten structures after global superposition. 

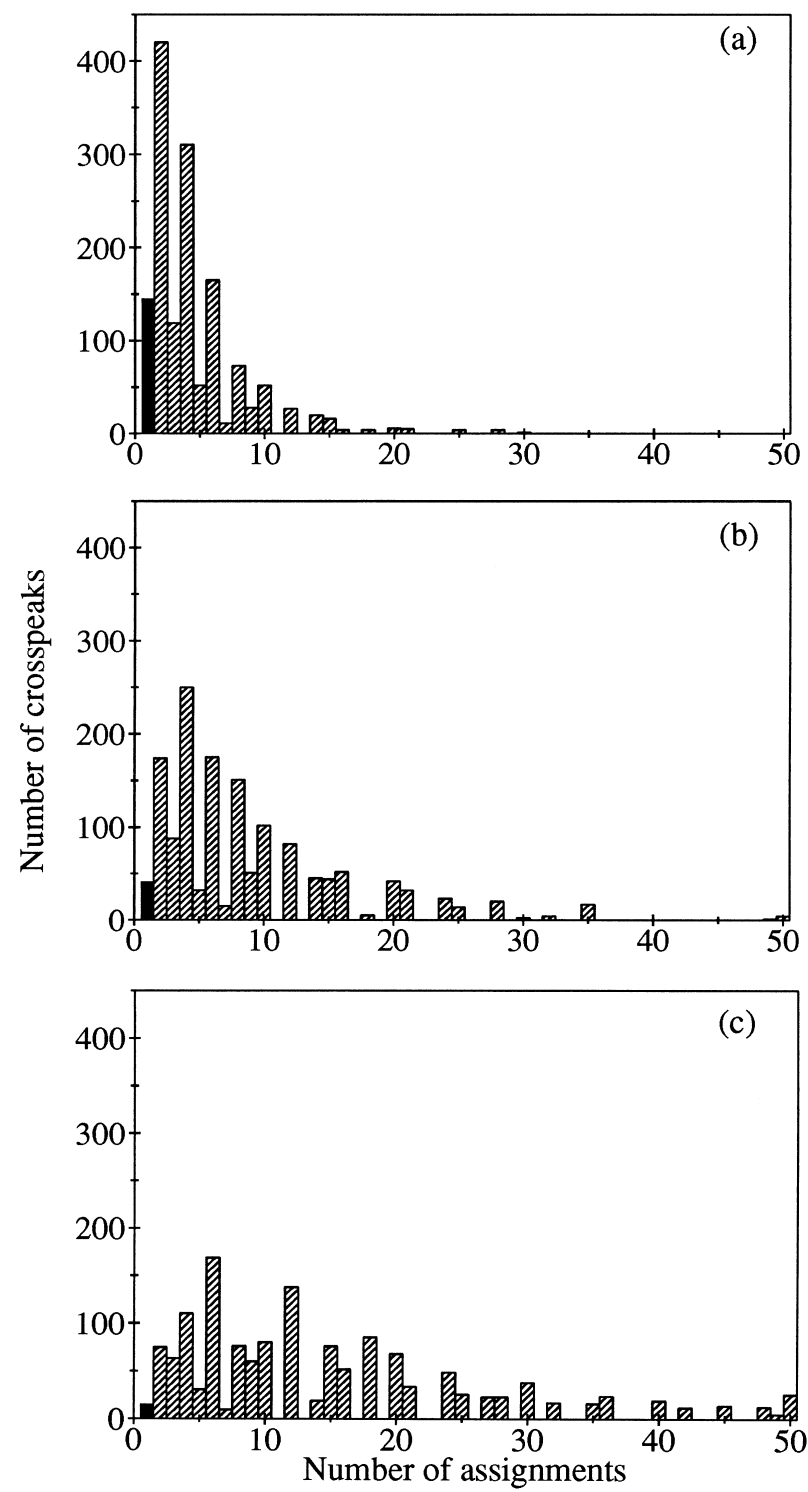

Figure 4. The histograms show the distribution of the NOE cross peaks with respect to the number of possible assignments made on the basis of chemical shifts alone. The data sets represented here are (a) DEN1, (b) DEN2 and (c) DEN3. The black bars highlight the cross peaks with unambiguous assignment.

unambiguously assigned peaks are plotted versus the NOAH cycles for the calculations DEN1, TEN1 and REP1. A particularly large increase in the number of these constraints can be observed after the sixth cycle. Towards the end of the calculations a saturation of the number of constraints was reached for all three proteins.

As the number of constraints increases, the bundle of calculated structures converges. The average pairwise DRMS values (Cohen \& Kuntz, 1989) of the ten structures with lowest target function value are shown in Figure 6. The DRMS values decrease considerably during the 25
Table 3. Violations of the distance constraints from the first NOAH cycle in the NMR reference structure

\begin{tabular}{|c|c|c|c|c|c|c|}
\hline \multirow[b]{2}{*}{ Code } & \multirow[b]{2}{*}{$N_{\text {con }}{ }^{\mathrm{a}}$} & \multirow{2}{*}{$\begin{array}{c}N_{\mathrm{ok}} \mathrm{b} \\
(\%)\end{array}$} & \multicolumn{4}{|c|}{ Number of violated constraints $\mathrm{c}$} \\
\hline & & & $0-3 \AA$ & $3-6 \AA$ & $6-9 \AA$ & $>9 \AA$ \\
\hline DEN1 & 962 & 63 & 56 & 60 & 66 & 173 \\
\hline DEN2 & 376 & 63 & 26 & 22 & 26 & 66 \\
\hline DEN3 & 158 & 66 & 15 & 6 & 7 & 26 \\
\hline DENa & 701 & 62 & 50 & 39 & 45 & 133 \\
\hline DENb & 393 & 62 & 26 & 27 & 28 & 70 \\
\hline TEN1 & 731 & 83 & 12 & 18 & 16 & 77 \\
\hline TEN2 & 361 & 65 & 24 & 31 & 24 & 46 \\
\hline REP1 & 600 & 72 & 22 & 28 & 28 & 92 \\
\hline REP2 & 183 & 71 & 7 & 6 & 6 & 34 \\
\hline NMRa & 794 & 58 & 93 & 52 & 58 & 130 \\
\hline $\mathrm{NMRb}$ & 1077 & 62 & 101 & 63 & 80 & 163 \\
\hline
\end{tabular}

a Total number of distance constraints.

${ }^{b}$ Percentage of the constraints that are consistent with the NMR reference structure.

${ }^{\mathrm{c}}$ Number of distance constraints that are violated in the NMR reference structure.

cycles, except in those cycles where certain test assignments were not included as constraints (see Methods).
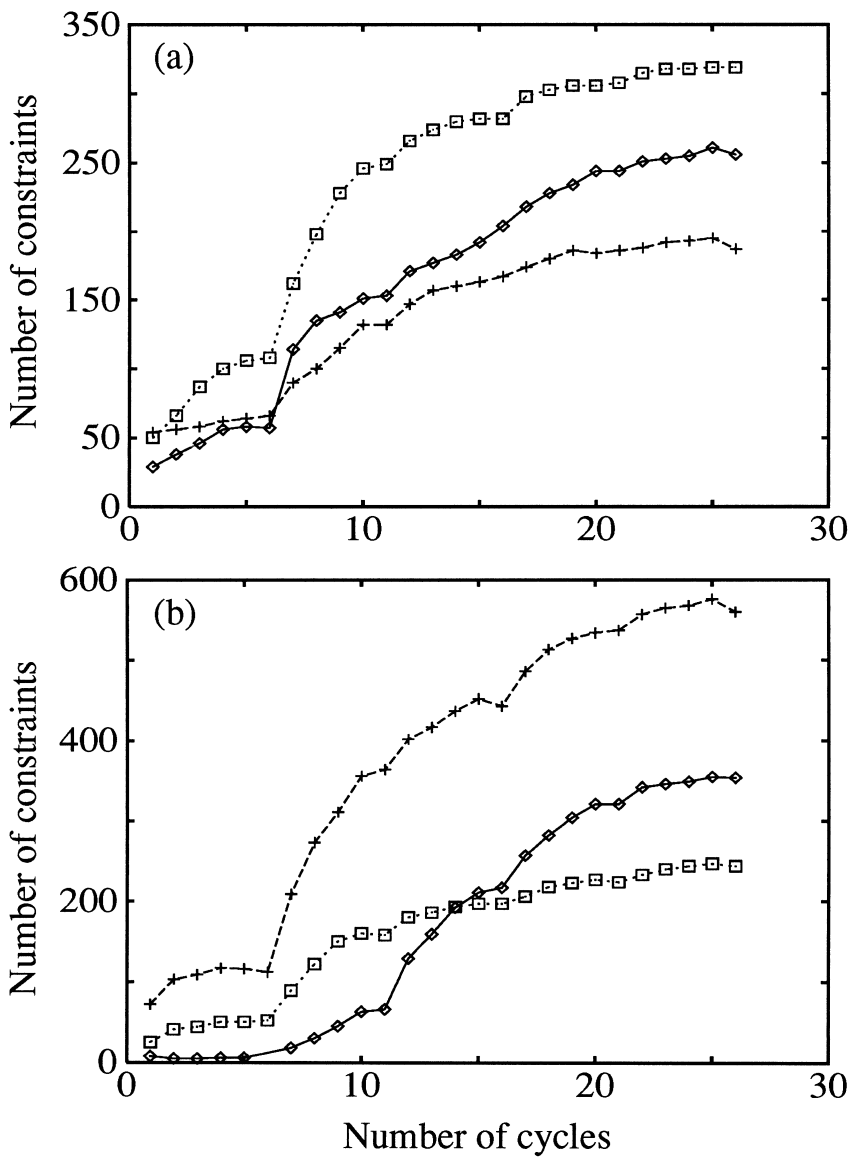

Figure 5. (a) Medium range and (b) long range distance constraints derived from the unambiguous assignment list (unambiguous AL) during the different NOAH cycles. The three curves correspond to the data set DEN1 (continuous line), TEN1 (broken line) and REP1 (dotted line). 


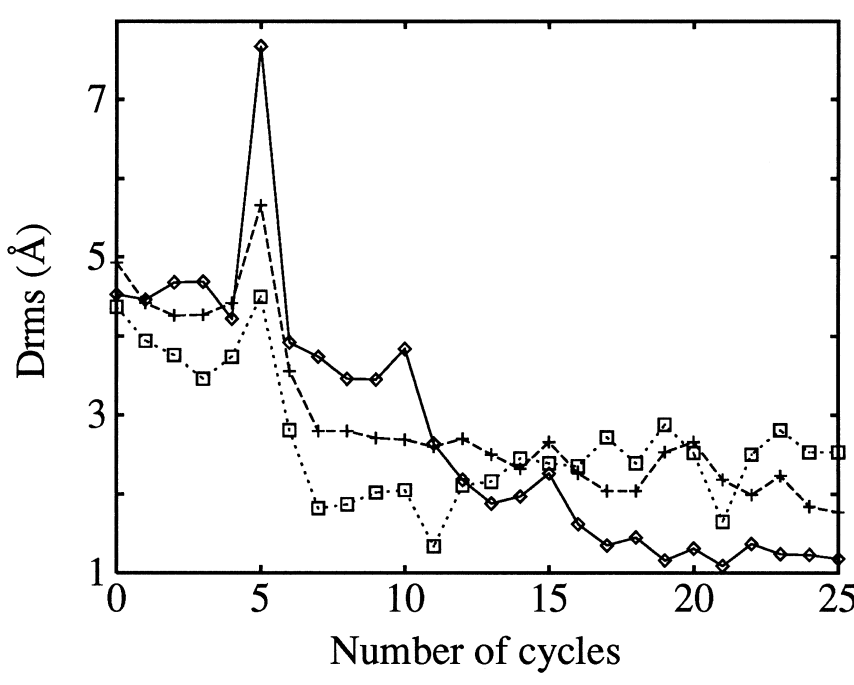

Figure 6. The decrease of the average DRMS between the structures during the NOAH cycles is illustrated for the data set DEN1 (continuous line), TEN1 (broken line) and REP1 (dotted line). Only the ten structures with the lowest target function values were considered and the DRMS was calculated for all $\mathrm{C}^{\alpha}$ atoms of the structures including flexible ends.

\section{Reliability check}

NOAH evaluates the reliability of the final assignments at the end of the calculations by two quantities, the number of inconsistent cross peaks $N_{\text {na }}$ and a reliability distance RD for each individual assignment. To calculate $N_{\text {na }}$, each unassigned cross peak is searched for possible assignments where the corresponding distance constraint is fulfilled by at least one of the calculated structures. If there is no such assignment, this unassigned cross peak is considered to be inconsistent with the calculated structures. $N_{\text {na }}$ counts the number of these inconsistent cross peaks. This number should be small if the final calculated structures form a narrow bundle around the correct structure. $N_{\text {na }}$ is listed for all the data sets in Table 4. The highest number is found in the calculation DEN3 where the final structure bundle is not well defined. In all other calculations, $N_{\text {na }}$ is less than $1 \%$ of the total number of cross peaks.

Secondly, the program calculates a reliability distance $\mathrm{RD}$ as additional information on all individual assigned peaks for the NMR experimentalist. A mathematical definition of the reliability distance is given in Methods. An RD of $5 \AA$ means that both protons would have to be moved by at least $5 \AA$ to fulfil any other assignment. An RD of $0 \AA$ means that with the given three-dimensional structures at least two assignments are possible within the chemical shift tolerance. In Table 4 we show that only a few incorrect assignments have an RD greater than $0 \AA$. The highest RD for wrongly assigned peaks correlated roughly with the pair- wise RMSD of the calculated structures. The maximum RD for a wrong constraint was always lower than or equal to $2.7 \AA$. All assignments made by NOAH with an RD above $3.0 \AA$ were correct in all calculations.

We used the reliability distance to calculate the theoretical limit of any structure based assignment method for NOESY spectra. We calculated the distribution of the reliability distance for the NMR reference structure and the complete list of correctly assigned peaks. Figure 7 shows these distributions for the three proteins dendrotoxin $\mathrm{K}$ (Figure 7(a)), tendamistat (Figure 7(c)) and P434 repressor (Figure 7 (e)). From 20 to $30 \%$ of all NOESY cross peaks have a reliability distance of $0 \AA$. These are peaks with two or more assignment possibilities within a chemical shift tolerance of $0.01 \mathrm{ppm}$ and uniform distance constraints of $5 \AA$.

The distribution of the reliability distance of the assigned peaks at the end of our calculations DEN1, TEN1 and REP1 with the ensemble of calculated structures is shown in Figure 7(b), (d) and (f), respectively. Almost all of the wrongly assigned peaks have a reliability distance of $0 \AA$ (black bars). The distribution of the RD values in our calculations is very similar to the distribution of the $\mathrm{RD}$ in the theoretical case. Especially the high numbers of critical assignment cases with reliability distance $0 \AA$ are similar to the theoretical cases. This shows that the final precision of our calculated structures is not the limiting factor for the accuracy of the assignment.

Table 4. Reliability checks

\begin{tabular}{|c|c|c|c|c|c|}
\hline \multirow[b]{2}{*}{ Code } & \multicolumn{2}{|c|}{ Unassigned peaks } & \multicolumn{3}{|c|}{ Assigned peaks } \\
\hline & $N_{\text {na }}{ }^{a}$ & $\begin{array}{c}R D_{\max }^{\mathrm{b}} \\
(\AA)\end{array}$ & $N_{>0} c^{\mathrm{c}}$ & $N_{\text {err }}{ }^{\mathrm{d}}$ & $\begin{array}{c}R D_{\max } \\
(\AA)\end{array}$ \\
\hline DEN1 & 0 & 0.0 & 1208 & 1 & 1.0 \\
\hline DEN2 & 3 & 1.1 & 720 & 3 & 0.4 \\
\hline DEN3 & 28 & 3.6 & 373 & 11 & 2.7 \\
\hline DENa & 7 & 0.9 & 1691 & 6 & 0.2 \\
\hline $\mathrm{DENb}$ & 14 & 1.2 & 847 & 11 & 1.0 \\
\hline TEN1 & 5 & 2.2 & 1286 & 0 & 0.0 \\
\hline TEN2 & 9 & 1.6 & 647 & 1 & 0.3 \\
\hline REP1 & 1 & 0.3 & 1109 & 0 & 0.0 \\
\hline REP2 & 1 & 2.2 & 672 & 0 & 0.0 \\
\hline NMRa & 8 & 1.7 & $644^{\mathrm{f}}$ & 0 & 0.0 \\
\hline $\mathrm{NMRb}$ & 12 & 3.4 & $654^{\mathrm{f}}$ & 0 & 0.0 \\
\hline
\end{tabular}

${ }^{a}$ Number of the remaining unassigned peaks which are incompatible with the final structure bundle. For these peaks, no distance constraint derived from all possible assignments is fulfilled in any of the ten structures.

${ }^{\mathrm{b}}$ Largest reliability distance value among the unassigned peaks.

${ }^{\mathrm{c}}$ Number of assigned peaks with a reliability distance above zero.

${ }^{d}$ Number of wrongly assigned peaks with a reliability distance above zero.

${ }^{\mathrm{e}}$ Highest reliability distance of a wrongly assigned peak. The values are given for $\Delta_{\text {tol }}=0.02 \mathrm{ppm}$ to account for the larger variations in the chemical shifts of the experimental data set. 

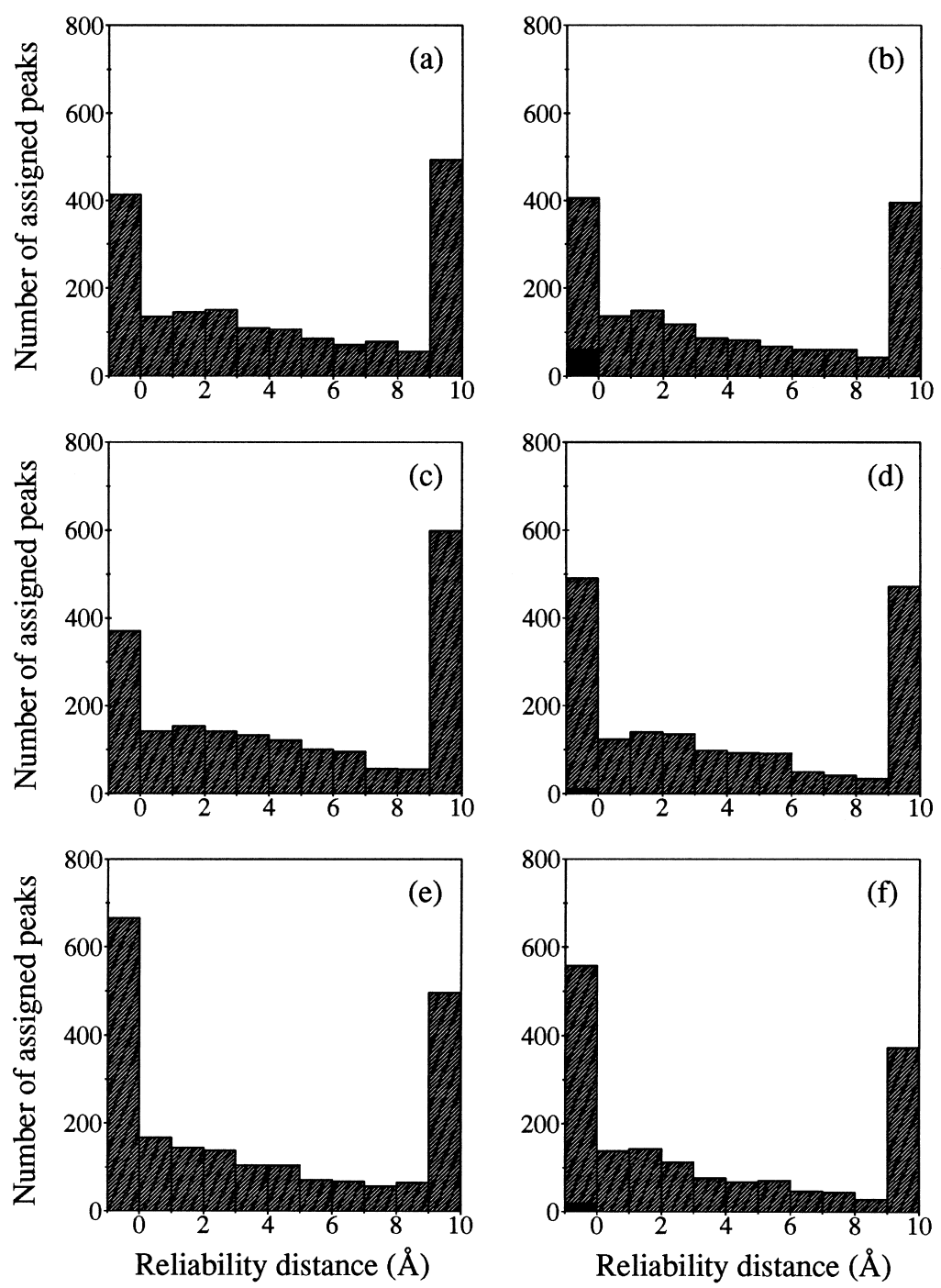

Figure 7. The histograms show the reliability distance (RD) distributions of the correct assignments ((a), (c) and (e)) and of the assignments obtained after the last NOAH cycle ((b), (d) and (f)) for the proteins dendrotoxin $\mathrm{K}$, tendamistat and repressor 434, respectively. We used the NMR reference structure to evaluate the theoretical RD distribution of the correct assignment and the bundle of structures after the last NOAH cycle for the distribution of the assignments made by NOAH. The RD was calculated with a chemical shift tolerance range $\Delta_{\text {tol }}$ of $0.01 \mathrm{ppm}$ and uniform distance constraints of $5 \AA$. The black bars mark the wrong peak assignments made by NOAH. All assignments with a RD above $10 \AA$ were added to the bar between 9 and $10 \AA$.

\section{Robustness to noise}

Our method is also robust if several hundreds of noise peaks are present in the peak list. We added 990 noise peaks on a grid in the range of $0 \mathrm{ppm}$ to $10 \mathrm{ppm}$ in both dimensions to the 1847 cross peaks of DEN1. Among the noise peaks there were 105 peaks with possible assignments within the given tolerance range $\Delta_{\text {tol }}=0.01 \mathrm{ppm}$. In a calculation with this peak list the final structures were only marginally influenced by the noise peaks and converged to the correct structure with a pairwise RMSD of $1.3 \AA$ and a RMSD of 1.2 to $1.6 \AA$ to the NMR reference structure. The assignment errors of $N_{\text {err }}=7.5 \%$ and the number of constraints violated in the NMR structure, $N_{\text {vio }}=1.6 \%$, were only slightly higher than in the DEN1 calculation (Table 2). Almost all of the 105 noise peaks, which have chemical shifts near observed proton chemical shifts, were not assigned, and only 12 noise peaks contributed to the assignment errors. In a second test calculation, where the number of noise peaks with possible assignments was increased to 210 , the final structures deviated significantly from the correct structures.

In both calculations NOAH warned the user that several hundreds of peaks had no possible assignments within the given tolerance range and that the number of inconsistent peaks, $N_{\text {na }}$, was high, i.e. 87 in the first calculation and 214 in the second calculation. For experimental data sets these cross peaks would have to be carefully examined by a line shape analysis to discard noise peaks (Antz et al., 1995).

\section{Discussion}

\section{Distance geometry with correct but incomplete data versus incorrect data}

Distance geometry methods are standard tools to calculate three-dimensional structures of proteins from NMR data. These methods use standard stereochemical properties of the amino acid residues and hard sphere models for non-bonded 
interactions. In the past the metric matrix distance geometry (Havel et al., 1983; Kuntz et al., 1989) and the variable target function method in torsion angles (Braun \& Gō, 1985; Braun, 1987; Güntert et al., 1991), both of them implemented in a variety of different programs, were mainly used. Due to spin diffusion and flexibility of the protein, the distance constraints were usually estimated only as upper limits, which form a correct but incomplete data set. The basic geometrical problem in the automated assignment of NOESY spectra is different. Incorrect distance constraints are included in the distance geometry calculations because of ambiguous assignments, leading to an ensemble of inconsistent constraints.

This type of problem can be solved by self-correcting distance geometry (Hänggi \& Braun, 1994; Mumenthaler \& Braun, 1995). This method tries to detect wrong constraints which are then eliminated in further calculations. Standard distance geometry programs are not suited for this problem, because they try to fulfil all constraints. In DIANA calculations with a test data set, including distance constraints which characterize an $\alpha$-helix (Wüthrich et al., 1984) with one inconsistent constraint, the final helices were significantly distorted, and the residual distance constraint violations were spread over a large number of constraints. In DIAMOD calculations with the same data set, only the inconsistent constraint showed large violations, and the backbone RMSD values of the DIAMOD structures to the $\alpha$-helix were much lower than in the DIANA structures.

\section{Accuracy and sampling of the calculated structures}

A major concern of our approach is the sampling property of the algorithm. In the past it has been extensively discussed (Wagner et al., 1987; Havel, 1990; Braun, 1991; Kuszewski et al.,1992; Nakai et al., 1993), whether distance geometry methods sufficiently sample all possible solution structures. By comparing structures calculated with the same data sets by different algorithms it was found that the methods used nowadays basically agree on the RMSD values.

This problem emerges again in distance geometry calculations with incorrect constraints. We have shown that the ten best structures selected by their final target function values cover the reference structures which were used to simulate the data (Table 2, Figure 2). The selection of the ten best structures can be justified by the $\mathrm{RMSD}_{\max }$ plot (Widmer et al., 1993) of the final structures (Figure 8).

Our procedure can also be applied if only few unambiguous long range cross peaks can be identified from their chemical shifts alone (Table 1). No initial preliminary three-dimensional structure is needed, which might bias the assignments in the first cycle. This is in contrast to the standard procedure, in which one first determines the global
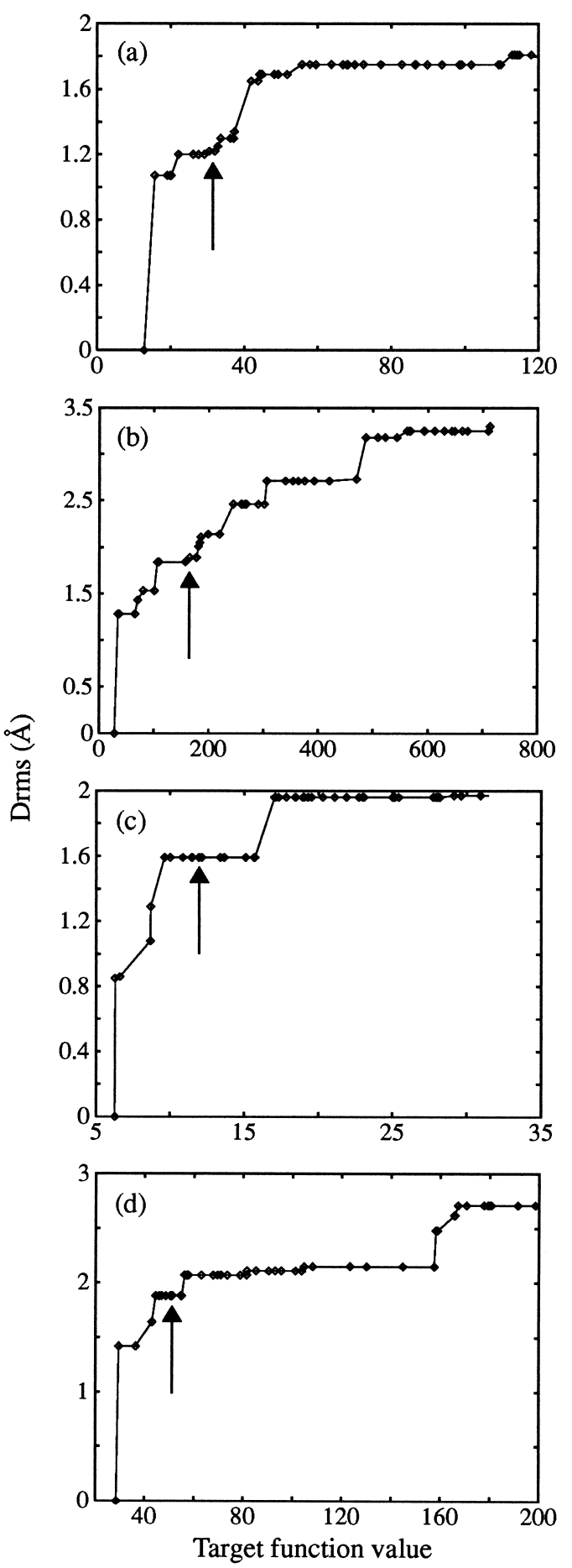

Figure 8. The maximum pairwise DRMS among the final structures is plotted versus the target function value for the data set (a) DEN1, (b) TEN1, (c) REP1 and (d) NMRa. The arrows point to the selected cutoffs of ten structures.

fold using only unambiguous cross peaks in a bootstrap technique (Braun, 1987; Güntert et al., 1993; Meadows et al., 1994). Our method has to overcome two potential difficulties: that the three-dimensional protein structures might never fold into compact structures or that they might converge towards a wrong fold "confirming" wrong 

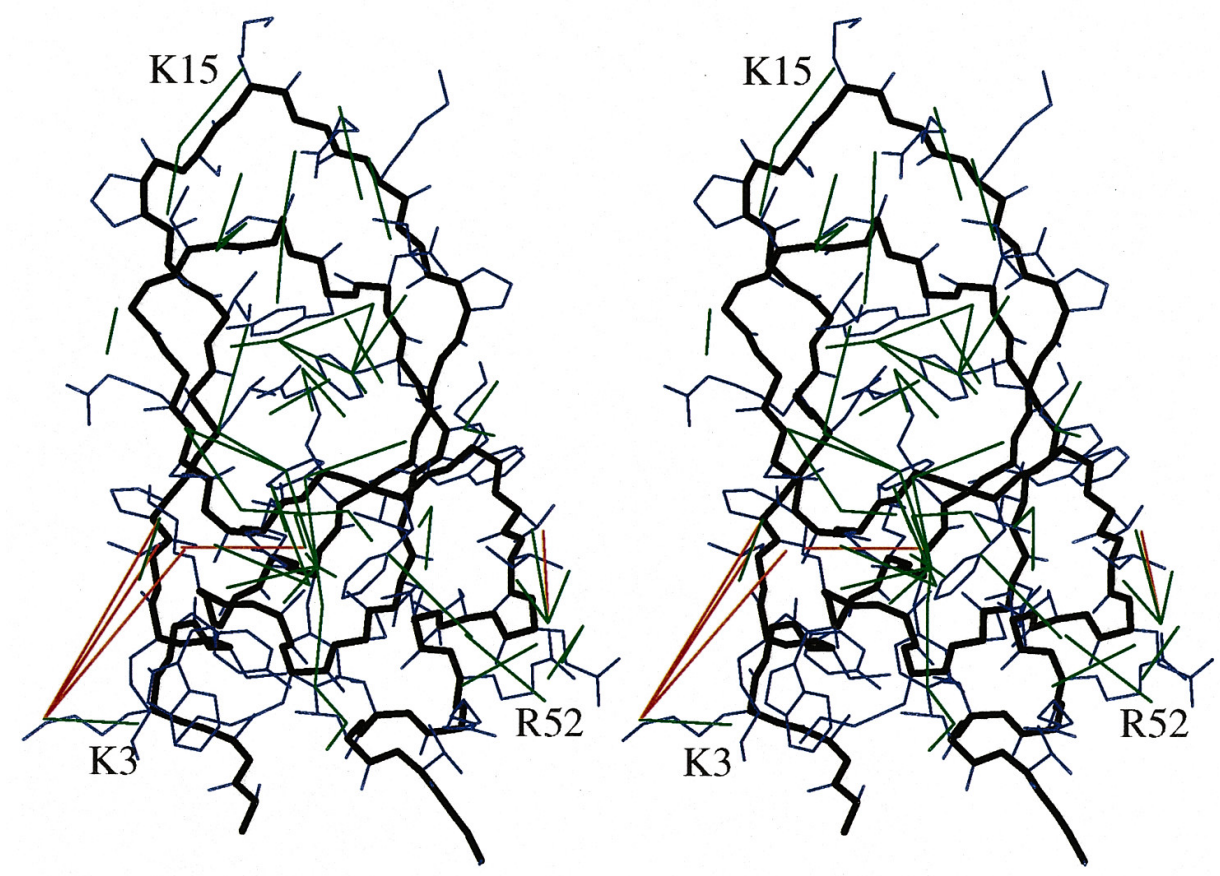

Figure 9. Stereoview of the NMR reference structure of dendrotoxin K (Berndt et al., 1993) where the distance constraints derived from all wrong assignments made in the calculation DEN1 are drawn as green and red lines. The green lines are constraints which are fulfilled in the NMR structure, the red lines are those constraints which are violated.

assignments made in the initial stage. When applied to three different proteins, our method successfully determined the backbone fold with an accuracy of about $1.5 \AA$. In practice, it should work for all small and medium size proteins with a cross peak list containing enough long range cross peaks. Currently the method has been applied only to homonuclear NOESY spectra, but it is straightforward to extend the procedure to heteronuclear 3D and 4D NOESY spectra (Clore \& Gronenborn, 1994).

\section{About the accuracy of the assignment}

Figure 9 shows all wrong assignments made in the calculation DEN1. The wrong assignments with a distance constraint not violated in the NMR reference structure are drawn as green lines. They are by far the majority of the wrong assignments. For such a wrongly assigned peak there must be another NOE cross peak within the assumed chemical shift tolerance, corresponding to this assignment. For structure determination these errors are not critical, as both NOESY cross peaks should be present. In practice both cross peaks appear as one composite peak in the NOESY spectrum, and the NOE intensity has to be separated into two components. We have not designed our method to treat especially this type of ambiguity, as was done by Nilges (1995). The analysis, however, shows that our method handles this type of ambiguity fairly well.

The red lines represent assignments which violate the NMR reference structure. There are only five violations and all of them are related to side-chain atoms of the surface residues K3, K24, K26 and R52. These errors are particularly difficult to detect because very few NOEs are usually observed in solvent exposed side-chains. Inconsistencies with other NOEs can therefore not be detected in these cases.

\section{Validation of the reliability checks}

A first critical check performed by NOAH tests the consistency of the calculated structures with all cross peaks in the peak lists. The assigned peaks are consistent with the calculated structures. For the unassigned peaks there are two possibilities: either there is more than one assignment possibility consistent with the calculated structures or no assignment within the chemical shift tolerance is compatible with the preliminary structures. There are several explanations for incompatible peaks: (1) the tolerance range $\Delta_{\text {tol }}$ is too small and the correct assignment was never considered. (2) The peak corresponds to a second possible protein conformation, e.g. different side-chain conformations in slow exchange (Otting et al., 1993). (3) The structure bundle deviates from the real structure. The possibility (1) can be eliminated by increasing the tolerance value $\Delta_{\text {tol }}$ and for possibility (2) there are usually additional peaks observed in J-correlated type spectra used in the sequential assignment procedure. If $N_{\text {na }}$ is still large compared to the total number of peaks $P_{\text {tot, }}$ the structures might have converged to a wrong fold. For this analysis we assumed that peaks from background noise and experimental artifacts had been removed to a large 
extent by other methods. As can be seen in Table 4 the number of inconsistent peaks $N_{\text {na }}$ was small in the calculations with simulated or experimental peak lists.

\section{Relation to other methods}

Others have studied the influence of subsets of distance constraints on the final structures with a completely different intent. With a cross-validation method this influence can be estimated and the completeness of the data sets can be checked (Brünger et al., 1993). This method is, however, not suited to detect inconsistent constraints. In the MEDUSA approach (Brüschweiler et al., 1991) one wants to study the conformational properties of an ensemble of interconverting structures of peptides, where no single conformation can satisfy all constraints (Dyson \& Wright, 1995). This procedure is computationally not feasible for proteins. Straightforward distance geometry calculations with different possibilities for assignments were used for some of the sequential assignments in melittin (Brown et al., 1982). This procedure is useful for small peptides to check the threedimensional consistencies of possible assignments, but it cannot be applied to proteins.

Kraulis assigned simulated peak lists by first calculating the three-dimensional positions of the unassigned protons before fitting the protein sequence into this "proton density map" (Kraulis, 1994). Good results were obtained for simulated peak lists containing 80 to $100 \%$ of all theoretically observable cross peaks within a distance of $4 \AA$. The RMSD of the average structure to the correct structure was around $1.5 \AA$. However, the calculations assumed that every cross peak could be unambiguously assigned to the correct proton pair from their chemical shifts. It remains to be seen to what extent experimental NOESY spectra of proteins with overlapping peaks can be assigned by this method.

More recently, Nilges could automatically assign simulated NOESY peak lists of the basic pancreatic trypsin-inhibitor (BPTI) by a completely different method (Nilges, 1995). All possible assignments of the cross peaks within a given chemical shift tolerance range $\Delta_{\text {tol }}$ are included in a $r^{-6}$ weighted sum during a single simulated annealing molecular dynamics run. Nilges used a different definition of the chemical shift tolerance $\Delta_{\text {tol }}$. He first rounded all chemical shifts to a multiple of $\Delta_{\mathrm{tol}}$, before selecting all possible assignments within a tolerance range of $+/-\Delta_{\text {tol }}$. His calculation N20 with a tolerance of $\Delta_{\text {tol }}=0.02 \mathrm{ppm}$ and $10.9 \%$ unambiguously assigned peaks, based on the chemical shift value alone, seems to correspond to our calculation with $\Delta_{\text {tol }}=0.01 \mathrm{ppm}$ and $9.9 \%$ unambiguously assigned peaks (DEN1). The histograms illustrating the number of possible assignments for the cross peaks, i.e. Figure 3(a) for data set N20 in his paper compared to Figure 4(a) or data set DEN1, support this assumption. Using this correspondence the performance of both methods are similar for simulated data sets.

\section{Methods}

\section{Hardware and software}

The program NOAH is written in FORTRAN 77. DIAMOD and NOAH calculations were run interleaved in a single shell script on a CRAY Y-MP. The CPU time needed for the 26 cycles was three to four hours for the data sets of Table 1 . The structures were analysed using software written in-house.

\section{Simulated NOESY cross peak lists}

The chemical shifts of the protons, the ${ }^{3} J_{\mathrm{NH} \alpha}$ coupling constants and the coordinates of the NMR solutions structures were extracted from the entries $1 \mathrm{dtk}$ for dendrotoxin K (Berndt et al., 1993), 2ait for tendamistat (Kline et al., 1988) and 1pra for the repressor 434 (Neri et al., 1992) of the Brookhaven Protein Data bank (Bernstein et al., 1977). We took the first structure from the PDB files 1dtk.pdb, 2ait.pdb and 1pra.pdb as reference structure for the NMR solution structures. We simulated peak lists by postulating one cross peak between all protons that are separated by less than a distance $d_{\text {sim }}=4 \AA$ for different data sets. Cross peaks were generated at the positions of the chemical shifts of the individual protons. In the different calculations these cross peak positions were only considered with a certain tolerance $\Delta_{\text {tol }}$ (Table 1$)$.

For DENa, a cutoff distance $d_{\text {sim }}=5.0 \AA$ was applied, yielding nearly twice the number of peaks compared to peak lists simulated with $d_{\text {sim }}=4.0 \AA$. In $\mathrm{DENb}$, peaks were randomly deleted from the DENa peak list until the size of the list generated with $d_{\text {sim }}=4.0 \AA$ was reached. $\mathrm{DENb}$ and DEN2 therefore contain the same number of peaks and have the same tolerance $\Delta_{\text {tol }}$. These data sets allow tests of the sensitivity of the method on the way the peak lists are simulated. Ideally, the method should give similar results for both of these data sets.

\section{Dihedral angle constraints}

The ${ }^{3} J_{\mathrm{NH} \alpha}$ coupling constants were translated into angle constraints for the $\Phi$ angles. For ${ }^{3} J_{\mathrm{NH} \alpha}$ smaller than $5.5 \mathrm{~Hz}$, $\Phi$ was restricted to the range $\left[-90^{\circ},-40^{\circ}\right]$. For ${ }^{3} J_{\mathrm{NH} \alpha}$ greater than $8.0 \mathrm{~Hz}, \Phi$ was restricted to $\left[-160^{\circ},-80^{\circ}\right]$ and for very large couplings greater than $10.0 \mathrm{~Hz}$, this range was narrowed to $\left[-140^{\circ},-100^{\circ}\right]$ (Kline et al., 1988). The number of angle restraints used in the calculations was 35 for dendrotoxin $\mathrm{K}, 44$ for tendamistat and 45 for the repressor 434 . The largest violation of such a constraint by the reference structures was $13^{\circ}$.

\section{The program NOAH}

The algorithm implemented in the program NOAH is described in Figure 10. No structures are available in the first cycle. NOAH determines the ambiguity of a cross peak by counting the number of possible assignments. Possible cross peak assignments are derived from the proton list with a certain tolerance $\Delta_{\text {tol }}$ in the chemical shifts. For a peak at position $\left(\omega_{1}, \omega_{2}\right)$ all protons with chemical shifts within the intervals $\left[\omega_{1}-\Delta_{\text {tol }}, \omega_{1}+\Delta_{\text {tol }}\right]$ 


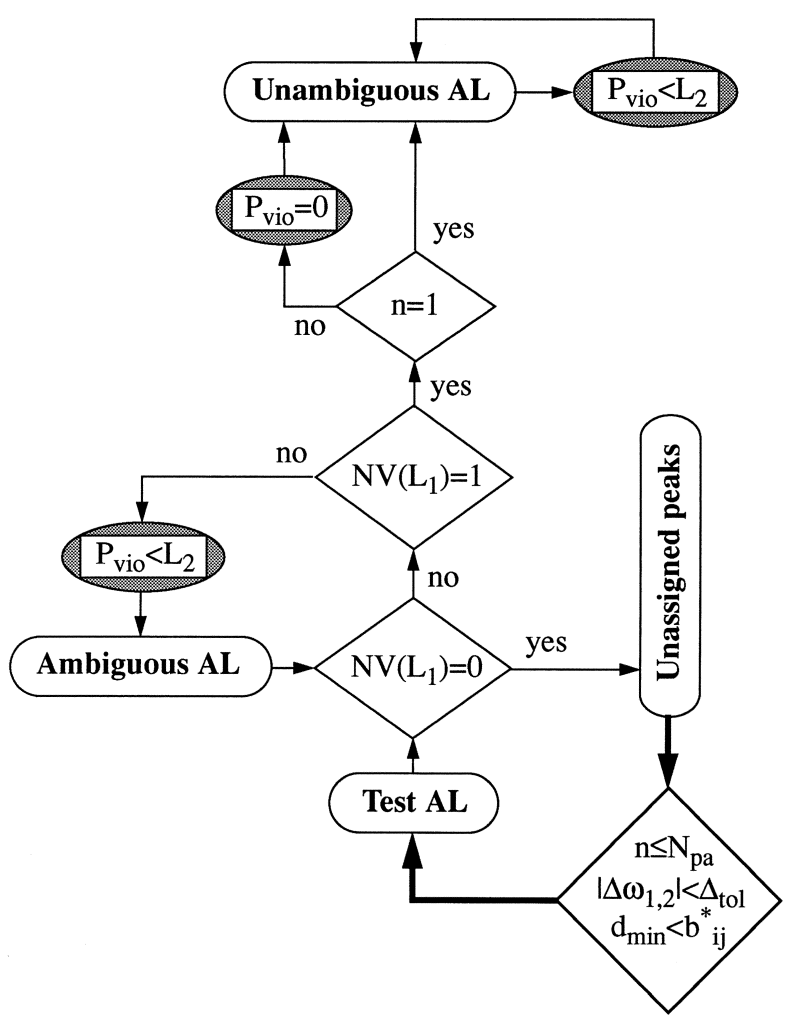

Figure 10. The different assignment lists (AL) used by NOAH are illustrated as well as all possible transition pathways. The bold arrow is the only transition which does not require preliminary structures. All other arrows show transitions induced by feedback filtering of the assignments through the structures. Filters are represented as grey discs with their criterion written in a white box. Only assignments which fulfil this criterion pass the filter. For every cross peak with $n$ possible assignments the corresponding $n$ distance constraints are analysed with respect to the ten preliminary structures with lowest target function. Every assignment has a $P_{\text {vio }}$ value which indicates the percentage of structures in which its constraint is violated. Every peak has a $N V(L)$ value which is the number of its distance constraints that are violated in less than $L \%$ of the preliminary structures.

and $\left[\omega_{2}-\Delta_{\text {tol }}, \omega_{2}+\Delta_{\text {tol }}\right]$ are considered. If the number of possible assignments is smaller than or equal to $N_{\mathrm{pa}}$, the assignments of this peak are stored in a test assignment list (test AL).

All test assignments are translated into upper limit distance constraints. For the simulated data sets a uniform distance constraint of $5.0 \AA$ was set with the standard pseudo-atom corrections: $2.4 \AA$ for QQD, QQG and QR atoms, $2.0 \AA$ for $\mathrm{CZ}$ and $\mathrm{CG}$ atoms, and $1.0 \AA$ for all other $\mathrm{Q}$ atoms (Wüthrich et al., 1983). For the experimental NMR data sets NMRa and NMRb, a $A \times r^{-6}$ calibration curve was used. The largest occurring peak volume was assumed to correspond to a distance of $2.5 \AA$ to determine the parameter $A$. This list of upper limit distances is then used as input for the distance geometry program DIAMOD which calculates an ensemble of three-dimensional structures.

In the following cycles the program NOAH uses these structures to generate new test assignments and to evaluate all assignments made in the previous cycle. As an additional criterion for including an assignment of a peak as a new test assignment, the program calculates the minimal distance $d_{\min }$ between the corresponding protons $i$ and $j$ in the $N$ structures with lowest target function values:

$$
d_{\min }=\min _{k=1, N}\left\{d_{i j}^{(k)}\right\}
$$

where $d_{i j}^{(k)}$ is the distance between the protons in the structure number $k . N=10$ was used in all of our calculations. The minimal distance $d_{\min }$ is compared to the corresponding upper distance constraint $b_{i j}$ for this assignment, and the program discards the assignment if $d_{\text {min }}>b_{i j}+d_{\text {tol }}=b_{i j}^{*}$. A tolerance distance $d_{\mathrm{tol}}$ is added to $b_{i j}$, because the preliminary structures calculated in the first few cycles may deviate from the correct solution structure.

To evaluate the assignments of the previous cycle, the program maintains two other assignment lists (Figure 10). The list of ambiguous assignments (ambiguous AL) contains peaks which have more than one possible assignment and the list of unambiguous assignments (unambiguous AL) contains peaks with a unique assignment. The list of unambiguous assignments is the result of the automatic assignment.

To filter all these assignment lists we calculate the parameter $P_{\text {vio }}$ :

$$
P_{\text {vio }}=\frac{1}{N} \sum_{k=1, N} \Theta\left(d_{i j}^{(k)}-b_{i j}-b_{t o l}\right)
$$

$P_{\text {vio }}$ gives the percentage of structures in which the distance constraint $b_{i j}$ plus a tolerance distance $b_{\text {tol }}$ is violated. $b_{\text {tol }}$ was set to $0.5 \AA$ in all calculations. $\Theta$ is the Heavyside function which is 0 at $x<0$ and 1 at $x \geqslant 0$. An additional variable, $N V(L)$, is introduced which counts the number of assignments of a specific peak that are violated by less than $L \%$ of the structures, i.e. where $P_{\text {vio }}<L \%$. An assignment is more likely to be correct if its distance constraint is violated only by a small number of structures. Assignments with $P_{\text {vio }}$ values below a certain threshold $\left(L_{1}\right)$, for example $40 \%$, are therefore classified as "probably correct", assignments with $P_{\text {vio }}$ values exceeding a second threshold $\left(L_{2}\right)$, for example $80 \%$, are "probably wrong".

If a peak has only one possible assignment $(n=1)$ and has a $P_{\text {vio }}$ value below $L_{1}\left(N V\left(L_{1}\right)=1\right)$, the peak is transferred to the list of unambiguous assignments. If a peak is ambiguous $(n>1)$, we have to distinguish three cases: (1) more than one assignment has a $P_{\text {vio }}$ value below $L_{1}\left(N V\left(L_{1}\right)>1\right)$. No definitive decision can then be made and the peak is transferred to the ambiguous assignment list with all its possible assignments that have a $P_{\text {vio }}$ below $L_{2}$. (2) Only one assignment has a value $P_{\text {vio }}$ lower than $L_{1}\left(N V\left(L_{1}\right)=1\right)$. If this best assignment has a $P_{\text {vio }}$ equal to $0 \%$, it is transferred to the unambiguous assignment list, because it is significantly better than the second best assignment by at least $L_{1}$. (3) All assignments have $P_{\text {vio }}$ values above $L_{1}\left(N V\left(L_{1}\right)=0\right)$. All proposed assignments are heavily violated by the structures. The peak is put back to the pool of unassigned peaks.

Furthermore, peaks from the unambiguous AL fall back into the pool of unassigned peaks if their assignment has become incompatible with the rest of the assignments (Figure 10). All assignments from the unambiguous, ambiguous and test assignment lists are translated into upper limit distance constraints in the previously described way. The constraints derived 
Table 5. NOAH parameters used in all calculations

\begin{tabular}{lcccc}
\hline Cycle & $L_{1}{ }^{\mathbf{a}}(\%)$ & $L_{2}{ }^{\mathbf{b}}(\%)$ & $N_{\mathrm{pa}^{\mathrm{c}}}$ & $d_{\mathrm{tol}^{\mathrm{d}}}(\AA)$ \\
\hline 0 & - & - & 2 & - \\
1 & 60 & 70 & 2 & 5.0 \\
2 & 50 & 70 & 2 & 4.0 \\
$3 \rightarrow 4$ & 40 & 70 & 2 & 4.0 \\
5 & 40 & 70 & - & - \\
$6-9$ & 40 & 70 & 2 & $3.0 \rightarrow 1.5$ \\
10 & 40 & 70 & - & - \\
$11 \rightarrow 14$ & 40 & 70 & 2 & 1.5 \\
15 & 40 & 70 & - & - \\
$16 \rightarrow 19$ & 40 & 70 & 3 & $1.5 \rightarrow 1.0$ \\
20 & 40 & 70 & - & - \\
$21 \rightarrow 24$ & 40 & 70 & 4 & 0.5 \\
25 & 40 & 70 & - & - \\
26 & 40 & 50 & - & - \\
\hline
\end{tabular}

a Assignments with a corresponding distance constraint that was violated by less than $L_{1} \%$ of the structures were classified as "probably correct".

b Assignments with a corresponding distance constraint that was violated by more than $L_{2} \%$ of the structures were classified as "probably wrong".

"Only cross peaks with a maximum of $N_{\text {pa }}$ possible assignments were used in the calculations.

d Tolerance distance used for the reduction of possible assignments through the preliminary structures. To check whether an assignment could eventually be fulfilled during the next structure calculations, the minimal distance $d_{\text {min }}$ between both protons in all structures is calculated. The assignment is only considered if $d_{\min }$ is smaller than the distance $b_{i j}+d_{\text {tol }}$ where $b_{i j}$ is the distance constraint given to that assignment.

from the unambiguous assignment list are weighted five times stronger than the constraints from the other peak lists.

\section{NOAH parameters used in the calculations}

The changes in the parameters $L_{1}, L_{2}, N_{\mathrm{pa}}$ and $d_{\mathrm{tol}}$ during the different NOAH cycles are listed in Table 5. An arbitrary number of 25 cycles was chosen for all calculations. An additional last NOAH cycle was performed to get the final assignments. The parameter $L_{1}$ was decreased from 60 to $40 \%$ in the first three cycles and stayed at $40 \%$ for all other cycles. $L_{2}$ was held constant (70\%) during the calculation and only decreased to $50 \%$ in the last cycle, just before the definitive assignment is produced by the program. $N_{\mathrm{pa}}$ increased from 2 to 4 during the calculations and $d_{\text {tol }}$ was decreased from 5 to $0.5 \AA$. Every fifth cycle, only constraints derived from the unambiguous and ambiguous assignment lists were used in the calculations. This yielded structure calculations with fewer but more accurate constraints, as these two lists contain only assignments that could be fulfilled by the structures in previous calculations.

During the first 25 DIAMOD cycles, only 40 structures were calculated each time and all hydrogen atoms were excluded in the van der Waals repulsion check. In the last cycle, 100 structures were calculated including all atoms.

\section{Reliability distance}

The program NOAH rates all proposed assignments by a reliability distance. For every peak, the program makes a list of all assignments that are possible based on the chemical shift tolerance $\Delta_{\text {tol }}$. For every assignment $k$, the minimal distance $d_{\min }(i, j)$ between the protons $i(k)$ and $j(k)$ in all $N$ structures is calculated as in formula (1). The number of assignments with corresponding constraints $b_{i j}$ that would be fulfilled by at least one structure are then counted with:

$$
F=\sum_{k=1, n} \Theta\left(b_{i j}-d_{\min }(i, j)\right)
$$

If $F$ is larger than 1 , the assignment is not very reliable, as at least one other alternative assignment is compatible with the structures and the reliability distance is set to $0 \AA$. In all other cases the reliability of the assignment can be defined as the distance to the next best assignment, i.e. to the assignment whose distance constraint has the smallest violation in any of the structures. If $k^{*}$ is the assignment of the given peak, we define the reliability distance RD by:

$$
\mathrm{RD}=\min _{\substack{k=1, n \\ k \neq k^{*}}}\left\langle d_{\min }(i, j)-b_{i j}\right\rangle
$$

A high RD for an assignment is a strong indication that it is correct, because both protons would have to be moved by the RD to fulfil the second best assignment possibility.

\section{Acknowledgements}

We acknowledge financial support to $\mathrm{Ch}$. M. by the ETHZ. We thank C. H. Schein for critical reading of the manuscript, and R. Koradi for use of the graphics software package MOLMOL. The use of the computing facilities of the IPS of the ETHZ is gratefully acknowledged.

\section{References}

Antz, Ch., Neidig, K. P. \& Kalbitzer, H. R. (1995). A general Bayesian method for an automated signal class recognition in 2D NMR spectra combined with a multivariate discriminant analysis. J. Biomol. NMR, 5, 287-296.

Arseniev, A. S., Kondakov, V. I., Maiorov, V. N. \& Bystrov, V. F. (1984). NMR solution spatial structure of "short" scorpion insectotoxin. FEBS Letters, 165, 57-62.

Bartels, Ch. (1995). Methoden der Zuordnung mehrdimensionaler magnetischer Kernspinresonanzspektren zur Strukturbestimmung von Makromolekülen. Ph D. Thesis, No 10966, ETH, Zürich, Switzerland.

Bax, A. \& Grzesiek, S. (1993). Methodological advances in protein NMR. Acc. Chem. Res. 26, 131-138.

Berndt, K. D., Güntert, P. \& Wüthrich, K. (1993). Nuclear magnetic resonance solution structure of dendrotoxin $\mathrm{K}$ from the venom of Dendroaspis polyepis polyepis. J. Mol. Biol. 234, 735-750.

Bernstein, F. C., Koetzle, T. F., Williams, G. J. B., Meyer, E. F. Jr, Brice, M. D., Rodgers, J. R., Kennard, O., Shimanouchi, T. \& Tasumi, M. (1977). The Protein Data Bank: a computer-based archival file for macromolecular structures. J. Mol. Biol. 112, 535-542.

Braun W. (1987). Distance geometry and related methods for protein structure determination from NMR data. Quart. Rev. Biophy. 19, 115-157.

Braun W. (1991). Distance geometry in torsion angle space: new developments and applications. In Computational Aspects of the Study of Biological Macromolecules by NMR (Hoch, J. C., Pulsen, F. M. \& Redfield, C., eds), pp. 199-208, Plenum Press, New York. 
Braun, W. \& Gō, N. (1985). Calculation of protein conformations by proton-proton distance constraints. J. Mol. Biol. 186, 611-626.

Braun, W., Bösch, C., Brown, L. R., Gō, N. \& Wüthrich, K. (1981). Combined use of proton-proton Overhauser enhancements and a distance geometry algorithm for determination of polypeptide conformations. Application to micelle-bound glucagon. Biochim. Biophys. Acta , 667, 377-396.

Braun, W., Wider, G., Lee, K. H. \& Wüthrich, K. (1983). Conformation of glucagon in a lipid-water interphase by ${ }^{1} \mathrm{H}$ nuclear magnetic resonance. J. Mol. Biol. 169, 921-948.

Braun, W., Wagner, G., Wörgötter, E., Vasak, M., Kägi, J. H. R. \& Wüthrich, K. (1986). Polypeptide fold in the two metal clusters of metallothionein-2 by nuclear nagnetic resonance and distance geometry. J. Mol. Biol. 187, 125-129.

Brown, L. R., Braun, W., Anil Kumar \& Wüthrich, K. (1982). High resolution nuclear magnetic resonance studies of the conformation and orientation of melittin bound to a lipid-water interface. Biophys. J. 37, 319-328.

Brünger, A. T., Clore, G. M., Gronenborn, A. M. \& Karplus, M. (1986). Three-dimensional structures of proteins determined by molecular dynamics with interproton distance restraints: application to crambin. Proc. Natl Acad. Sci. USA, 83, 3801-3805.

Brünger, A. T., Clore, G. M., Gronenborn, A. M., Saffrich, R. \& Nilges, M. (1993). Assessing the quality of solution nuclear magnetic resonance structures by complete cross-validation. Science, 261, 328-331.

Brüschweiler, R., Blackledge, M. \& Ernst, R. R. (1991). Multi-conformational peptide dynamics derived from NMR data: A new search algorithm and its application to antamanide. J. Biomol. NMR, 1, 3-11.

Clore, G. M. \& Gronenborn, A. M. (1991). Structures of larger proteins in solution: three- and fourdimensional heteronuclear NMR spectroscopy. Science, 252, 1390-1399.

Clore, G. M. \& Gronenborn, A. M. (1994). Multidimensional heteronuclear nuclear magnetic resonance of proteins. Methods Enzymol. 239, 349-363.

Clore, G. M., Gronenborn, A. M., Brünger, A. T. \& Karplus, M. (1985). Solution conformation of a heptadecapeptide comprising the DNA binding helix $\mathrm{F}$ of the cyclic AMP receptor protein of Escherichia coli. Combined use of ${ }^{1} \mathrm{H}$ nuclear magnetic resonance and restrained molecular dynamics. J. Mol. Biol. 186, 435-455.

Clore, G. M., Robien, M. A. \& Gronenborn, A. M. (1993). Exploring the limits of precision and accuracy of protein structures determined by nuclear magnetic resonance spectroscopy. J. Mol. Biol. 231, 82-102.

Cohen, F. E. \& Kuntz, I. D. (1989). Tertiary structure prediction. In Prediction of Protein Structure and the Principles of Protein Conformation (Fasman, G. D., ed.), pp. 647-705, Plenum Press, New York.

Dyson, H. J. \& Wright, P. E. (1995). Antigenic peptides. FASEB J. 9, 37-42.

Fairbrother, W. J., Gippert, G. P., Reizer, J., Saier, M. H. \& Wright, P. E. (1992). Low resolution solution structure of the Bacillus subtilis glucose permease IIA domain derived from heteronuclear three-dimensional NMR spectroscopy. FEBS Letters, 296, 148-152.

Fesik, S. W. \& Zuiderweg, E. R. P. (1988). Heteronuclear three-dimensional NMR spectroscopy. A strategy for the simplification of homonuclear two-dimensional NMR spectra. J. Magn. Reson. 78, 588-593.
Fogh, R. H., Schipper, D., Boelens, R. \& Kaptein, R. (1994). ${ }^{1} \mathrm{H},{ }^{13} \mathrm{C}$, and ${ }^{15} \mathrm{~N}$ NMR backbone assignments of the 269-residue serine PB92 from Bacillus alcalophilus. I. Biomol. NMR, 4, 123-128.

Gronenborn, A. M. \& Clore, G. M. (1994). Where is NMR taking us? Proteins: Struct. Funct. Genet. 19, 273-276.

Grzesiek, S., Döbeli H., Genz, R., Garotta, G., Labhardt, A. M. \& Bax, A. (1992). ${ }^{1} \mathrm{H},{ }^{13} \mathrm{C}$, and ${ }^{15} \mathrm{~N}$ NMR backbone assignments and secondary structure of human interferon- $\gamma$. Biochemistry, 31, 8180-8190.

Güntert, P., Braun, W., Billeter, M. \& Wüthrich, K. (1989). Automated stereospecific ${ }^{1} \mathrm{H}$ NMR assignments and their impact on the precision of protein structure determination in solution. J. Am. Chem. Soc. 111, 3997-4004.

Güntert, P., Braun, W. \& Wüthrich, K. (1991). Efficient computation of three-dimensional protein structures in solution from NMR data using the program DIANA and the supporting programs CALIBA, HABAS, and GLOMSA. J. Mol. Biol. 217, 517-530.

Güntert, P., Berndt, K. D. \& Wüthrich, K. (1993). The program ASNO for computer-supported collection of NOE upper distance constraints as input for protein structure determination. J. Biomol. NMR, 3, 601-606.

Hänggi, G. \& Braun, W. (1994). Pattern recognition and self-correcting distance geometry calculations applied to myohemerythrin. FEBS Letters, 344, 147-153.

Hare, B. J. \& Prestegard, J. H. (1994). Application of neural networks to automated assignment of NMR spectra of proteins. J. Biomol. NMR, 4, 35-46.

Havel, T. F. (1990). The sampling properties of some distance geometry algorithms applied to unconstrained polypeptide chains: a study of 1830 independently computed conformations. Biopolymers, 29, 1565-1585.

Havel, T. F., Kuntz, I. D. \& Crippen, G. M. (1983). The theory and practice of distance geometry. Bull. Math. Biol. 45, 665-720.

Kaptein, R., Zuiderweg, E. R. P., Scheek, R. M., Boelens, R. \& van Gunsteren, W. F. (1985). A protein structure from nuclear magnetic resonance data. lac repressor headpiece. J. Mol. Biol. 182, 179-182.

Kline, A. D., Braun, W. \& Wüthrich, K. (1986). Studies by ${ }^{1} \mathrm{H}$ nuclear magnetic resonance and distance geometry of the solution conformation of the $\alpha$-amylase inhibitor Tendamistat. J. Mol. Biol. 189, 377-382.

Kline, A. D., Braun, W. \& Wüthrich, K. (1988). Determination of the complete three-dimensional structure of the $\alpha$-amylase inhibitor tendamistat in aqueous solution by nuclear magnetic resonance and distance geometry. J. Mol. Biol. 204, 675-724.

Kraulis, P. J. (1994). Protein three-dimensional structure determination and sequence-specific assignment of ${ }^{13} \mathrm{C}$ and ${ }^{15} \mathrm{~N}$-separated NOE data. A novel real-space ab initio approach. J. Mol. Biol. 243, 696-718.

Kuntz, I. D., Thomason, J. F. \& Oshiro, C. M. (1989). Distance geometry. Methods Enzymol. 177, 159-204.

Kuszewski, J., Nilges, M. \& Brünger, A. T. (1992). Sampling and efficiency of metric matrix distance geometry: A novel partial metrization algorithm. J. Biomol. NMR, 2, 33-56.

Meadows, R. P., Olejniczak, E. T. \& Fesik, S. W. (1994). A computer-based protocol for semiautomated assignments and 3D structure determination of proteins. J. Biomol. NMR, 4, 79-96.

Morelle, N., Brutscher, B., Simorre, J.-P. \& Marion, D. (1995). Computer assignment of the backbone resonances of labelled proteins using two- 
dimensional correlation experiments. J. Biomol. NMR, 5, 154-160.

Mumenthaler, Ch. \& Braun, W. (1995). Predicting the helix packing of globular proteins by self-correcting distance geometry. Protein Sci. 4, 863-871.

Nagayama, K. \& Wüthrich, K. (1981). Systematic application of two-dimensional ${ }^{1} \mathrm{H}$ nuclear-magneticresonance techniques for studies of proteins. Eur. J. Biochem. 114, 365-374.

Nakai, T., Kidera, A. \& Nakamura, H. (1993). Intrinsic nature of the three-dimensional structure of proteins as determined by distance geometry with good sampling properties. J. Biomol. NMR, 3, 19-40.

Neri, D., Billeter, M. \& Wüthrich, K. (1992). Determination of the nuclear magnetic resonance solution structure of the DNA-binding domain (residues 1 to 69) of the 434 repressor and comparison with the X-ray crystal structure. J. Mol. Biol. 223, 743-767.

Nilges, M. (1995). Calculation of protein structures with ambiguous distance restraints. Automated assignment of ambiguous NOE crosspeaks and disulphide connectivities. J. Mol. Biol. 245, 645-660.

Olson, J. B. Jr \& Markley, J. L. (1994). Evaluation of an algorithm for the automated sequential assignment of protein backbone resonances: a demonstration of the connectivity tracing assignment tools (CONTRAST) software package. J. Biomol. NMR, 4, 385-410.

Oshiro, C. M. \& Kuntz, I. D. (1993). Application of distance geometry to the proton assignment problem. Biopolymers, 33, 107-115.

Otting, G., Liepinsh, E. \& Wüthrich, K. (1993). Disulfide bond isomerization in BPTI and BPTI(G36S): an NMR study of correlated mobility in proteins. Biochemistry, 32, 3571-3582.

Remerowski, M. L., Domke, T., Groenewegen, A., Pepermans, H. A. M., Hilbers, C. W. \& van de Ven, F. J. M. (1994). ${ }^{1} \mathrm{H},{ }^{13} \mathrm{C}$, and ${ }^{15} \mathrm{~N}$ NMR backbone assignments and secondary structure of the 269residue protease subtilisin 309 from Bacillus lentus. J. Biomol. NMR, 4, 257-278.

Spitzfaden, C., Braun, W., Wider, G., Widmer, H. \& Wüthrich, K. (1994). Determination of the NMR solution structure of the cyclophilin-cyclosporin A complex. J. Biomol. NMR, 4, 463-482.

Stockmann, B. J., Nirmala, N. R., Wagner, G., Delcamp, T. J., De Yarman, M. T. \& Freisheim, J. H. (1992). Sequence-specific ${ }^{1} \mathrm{H}$ and ${ }^{15} \mathrm{~N}$ resonance assignments for human dihydrofolate reductase in solution. Biochemistry, 31, 218-229.
Thériault, Y., Logan, T. M., Meadows, R., Yu, L., Olejniczak, E. T., Holzmann, T. F., Simmer, R. L. \& Fesik, S. W. (1993). Solution structure of the cyclosporin A/cyclophilin complex by NMR. Nature, 361, 88-91.

Wagner, G. (1993). Prospects for NMR of large proteins. J. Biomol. NMR, 3, 375-385.

Wagner, G. \& Wüthrich, K. (1982). Sequential resonance assignments in protein ${ }^{1} \mathrm{H}$ nuclear magnetic resonance spectra. Basic pancreatic trypsin inhibitor. J. Mol. Biol. 155, 347-366.

Wagner, G., Braun, W., Havel, T. F., Schaumann, T., Gō, N. \& Wüthrich, K. (1987). Protein structures in solution by nuclear magnetic resonance and distance geometry: the polypeptide fold of the basic pancreatic trypsin inhibitor determined using two different algorithms, DISGEO and DISMAN. J. Mol. Biol. 196, 611-641.

Widmer, H., Widmer, A. \& Braun, W. (1993). Extensive distance geometry calculations with different NOE calibrations. New criteria for structure selection applied to sandostatin and BPTI. J. Biomol. NMR, 3, 307-324.

Williamson, M. P., Havel, T. F. \& Wüthrich, K. (1985). Solution conformation of proteinase inhibitor IIA from bull seminal plasma by ${ }^{1} \mathrm{H}$ nuclear magnetic resonance and distance geometry. J. Mol. Biol. 182, 295-315.

Wüthrich, K., Wider, G., Wagner, G. \& Braun, W. (1982). Sequential resonance assignments as a basis for determination of spatial protein structures by high resolution proton nuclear magnetic resonance. J. Mol. Biol. 155, 311-319.

Wüthrich, K., Billeter, M. \& Braun, W. (1983). Pseudostructures for the 20 common amino acids for use in studies of protein conformations by measurements of intramolecular proton-proton distance constraints with nuclear magnetic resonance. J. Mol. Biol. 169, 949-961.

Wüthrich, K., Billeter, M. \& Braun, W. (1984). Polypeptide secondary structure determination by nuclear magnetic resonance observation of short proton-proton distances. J. Mol. Biol. 180, 715740.

Zimmermann, D., Kulikowski, C., Wang, L., Lyons, B. \& Montelione, G. T. (1994). Automated sequencing of amino acid spin systems in proteins using multidimensional HCC(CO)NH-TOCSY spectroscopy and constraint propagation methods from artificial intelligence. J. Biomol. NMR, 4, 241-256.

Edited by P. E. Wright

(Received 11 July 1995; accepted 11 September 1995) 